\title{
Improved Krill Herd Algorithm with Neighborhood Distance Concept for Optimization
}

\author{
Pras un Kumar Agrawal ${ }^{1}$, Manjaree Pandit ${ }^{1}$, Hari Mohan Dube ${ }^{* 1}$ \\ Department of Electrical Engineering, ${ }^{1}$ M.I.T.S, Gwalior, India \\ *Corresponding Author, Tel.+91-0751-2665962, +91-0751-2409348, E-mail: harimohandubeymits @ gmail.com
}

\begin{abstract}
Krill herd algorithm (KHA) is a novel nature inspired (NI) optimization technique that mimics the herding behavior of krill, which is a kind of fish found in nature. The mathematical model of KHA is based on three phenomena observed in the behavior of a herd of krills, which are, moment induced by other krill, foraging motion and random physical diffusion. These three key features of the algorith $\mathrm{m}$ provide a good balance between global and local search capability, which makes the algorith $m$ very powerful. The objective is to minimize the distance of each krill from the food source and also from the point of highest density of the herd, which helps in convergence of population around the food source. Improvisation has been made by introducing neighborhood distance concept along with genetic reproduction mechanism in basic $\mathrm{KH}$ Algorithm. KHA with mutation and crossover is called as (KHAMC) and KHA with neighborhood distance concept is referred here as (KHAMCD). This paper compares the performance of the KHA with its two improved variants KHAMC and KHAMCD. The performance of the three algorithms is compared on eight benchmark functions and also on two real world economic load dispatch (ELD) problems of power system. Results are also compared with recently reported methods to establish robustness, validity and superiority of the KHA and its variant algorithms.
\end{abstract}

Index Terms-Krill Herd Algorithm (KHA), mutation and crossover, neighborhood distance concept, unimodal function, multimodal function, economic load dispatch.

\section{INTRODUCTION}

Optimization is basically a spontaneous process that plays an important role in real world application. Its objective is to compute a set of variables that either minimize or maximize the objectives function within given constraints. For solution of a given model or an objective function, there is a need of efficient optimization techniques which can either be conventional (deterministic) or have a stochastic, evolutionary approach. Conventional techniques include nonlinear programming, linear programming, quadratic programming, Newton's method etc. Deterministic approaches generally require an in itial guess which has a vital impact on the final solution. Considering practical utility of optimization there is need of robust and efficient algorithms which are free from this limitation.
Generally the practical problems are much complex and also have many constraints which cannot be solved by conventional approaches. On the other hand, nature inspired methods which are basically population based stochastic search techniques often provides quick and reasonable solution; though a careful tuning of parameters is required to prevent solution from getting trapped in local minima. In the recent years various population based evolutionary techniques have been developed for solution of problems related to real world application.

Among evolutionary algorith ms genetic algorithm (GA) is probably the most popular algorithm based on Darwinian evolution concept proposed by Holland in 1992[1]. Simple concepts are involved in it and involvement of stochastic operators may be the key point for popularity of this algorithm. After GA various nature inspired algorithms have been proposed such as Particle Swarm Optimization(PSO)[7], Ant Colony Optimization(ACO)[8], Harmony Search Algorithm (HSA)[9], Artificial Bee Colony Algorithm(ABC)[15], Gravitational Search Algorithm(GSA)[16] etc, which may be based on natural concepts of evolution, collective behavior, ecology or physical science [2-26] listed in Table 1. Each algorithm has its own advantage. But the key points associated with evolutionary algorith ms which make them popular for solution of complex constrained problem in comparison to conventional approach are depicted in Table 2. In fact there is no optimization technique has been developed that can capable to solve all types of optimization problems [27]. A comparative study of NI algorithms for unimodal and multimodal optimization problem is presented in ref. [55].

Among NI algorithms krill herd algorithm (KHA) is novel optimization techniques that inspired by herding behavior of krill herd. KHA implemented to solve different types of real world optimization problems either by hybridizing with other evolutionary algorithm to improve the basic KHA or by adding some mathematical concept [28-38]. Variant of KHA proposed till date is depicted in Fig. 1. In this paper KHA with neighborhood distance concept is proposed and their performances were analyzed using benchmark functions and practical complex constrained problems related to economic load dispatch (ELD) of power system.

This paper organized as below: section I deals with introduction to optimization techniques, section II presents krill herd algorithm and its variants. The 
performance evaluation on numerical benchmarks and using standard complex constrained test cases of ELD are presented in section III and IV respectively. Finally concluding remarks are presented in section $\mathrm{V}$.

Table 1. Development of Nature Inspired (NI) Algorithms in Chronological Order

\begin{tabular}{|l|l|l|l|}
\hline Year & Name & Year & Name \\
\hline 1966 & Evolution Strategies [2] & 2007 & Firefly Algorithm [14] \\
\hline 1966 & Evolutionary Programming [3] & 2007 & Artificial Bee Colony Algorithm [15] \\
\hline 1975 & Genetic Algorithms [1] & 2009 & Gravitational Search Algorithm [16] \\
\hline 1979 & Cultural Algorithms [4] & 2010 & Bat Algorithm [17] \\
\hline 1983 & Simulated Annealing [5] & 2011 & Cuckoo Search Algorithm [18] \\
\hline 1989 & Tabu Search [6] & 2012 & Krill Herd Algorithm [19] \\
\hline 1995 & Particle Swarm Optimization [7] & 2013 & Social Spider optimization [20] \\
\hline 1996 & Ant Colony Optimization [8] & 2013 & Backtracking Search Algorithm [21] \\
\hline 2001 & Harmony Search Algorithm [9] & 2014 & Grey Wolf Optimization [22] \\
\hline 2002 & Estimation of Distribution Algorithm [10] & 2014 & Symbiotic organism Search Algorithm [23] \\
\hline 2002 & Bacterial Foraging Algorithm [11] & 2015 & Lion Optimization Algorithm [24] \\
\hline 2005 & Honey Bee Mating Optimization Algorithm [12] & 2015 & Stochastic fractal Search [25] \\
\hline 2007 & Intelligent Water Drops [13] & 2015 & Lightening Search [26] \\
\hline
\end{tabular}

Table 2. Comparison of Traditional \& Evolutionary Technique

\begin{tabular}{|l|l|}
\hline \multicolumn{1}{|c|}{ Traditional Technique } & Evolutionary Technique \\
\hline Single point search & Population based search \\
\hline Gradient search & Random search \\
\hline Deterministic algorithm & Stochastic algorithm \\
\hline Mathematical principle & Natural \& physical based \\
\hline Fast but will not run for many problems. & $\begin{array}{l}\text { Slow but run for most of the real world problem, } \\
\text { complex and undefined problems. }\end{array}$ \\
\hline Same solution every time & Different solution with time accuracy. \\
\hline Different solver required for different function. & Independent of objective functions. \\
\hline
\end{tabular}

\section{KRILL HERD ALGORITHM AND ITS MODULES}

Krill is a species of fish which are generally found in oceans. Based on the breeding mechanism, they have the ability to form large swarm population. KHA mimics the collective behavior of krill swarm considering their selfposition as well as their position in group. During optimization the global optima solution refers the minimum distance of a krill individual from highest density food source whereas individual krill try to migrate near the best solution.

\section{A. Lagrangian model of Krill Herd Algorithm (KHA)}

The fitness function combines position of individual krill from food source and density of krill around the food source. The movement of each krill in two dimensional search spaces can be evaluated on the bas is of movement of other krill, foraging activity and the physical diffusion process.

\section{1) Motion induced by other krill individual}

Maintaining high density is essential to get optimal solution in KHA because the fitness function highly influenced by density of krill in search space. The direction of krill movement is computed on the basis of local swarm density, target swarm density and repulsive swarm density as it highly influenced by neighboring krill. Considering all effects simultaneously velocity of movement for $i^{t h}$ krill can be expressed as [19, 46]:

$$
v_{i}^{\text {new }}=v^{\max } \sigma_{i}+\omega_{n} \times v_{i}^{\text {old }}
$$

Where $v^{\max }$ are the motion induced by other krill, $v_{i}^{\text {new }} \&$ $v_{i}^{\text {old }}$ are the motion induced by $\mathrm{i}^{\text {th }}$ krill at modified as well their previous movement and $\omega_{n}$ is the inertia weight for induced motion. 
The direction of motion $\left(\sigma_{i}\right)$ of $i^{\text {th }}$ krill can be expressed as:

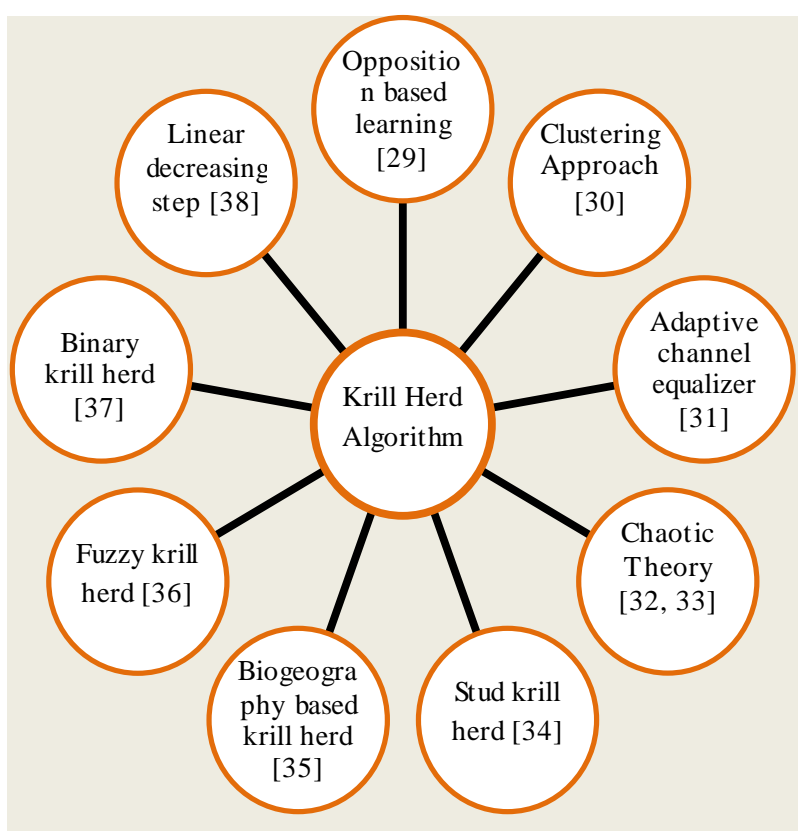

Fig.1. Variants of Krill Herd Algorithm

$$
\sigma_{i}=\sum_{j=1}^{n b}\left[\frac{f_{i}-f_{j}}{f^{\text {worst }}-f^{\text {best }}} \times \frac{K_{j}-K_{i}}{\left|K_{j}-K_{i}\right|+\operatorname{rand}(0,1)}\right]+2 \times\left[\operatorname{rand}(0,1)+\frac{i \text { iter }}{\text { iter }}{ }^{\max }\right] f_{i}^{\text {best }} x_{i}^{\text {best }}
$$

Where $f_{i}, f_{j}$ are the fitness of $i^{\text {th }}$ and $j^{\text {th }} \mathrm{krill}$, $f_{i}^{\text {worst }}, f_{i}^{\text {best }}$ are the worst and best fitness among krill swarm and $n b$ is the total number of neighbor.

Selection of neighbor is based on the distance senesced $(d s)$ by each krill, it is defined as:

$$
d s_{i}=\frac{i}{5 N} \sum_{k=1}^{N}\left|K_{i}-K_{k}\right|
$$

Where $N$ is the number of krill (population size) and $\mathrm{K}_{\mathrm{i}}$, $\mathrm{K}_{\mathrm{k}}$ are the position of $i^{\text {th }}$ and $k^{\text {th }}$ of krill respectively.

\section{2) Foraging motion}

Movement of krill individual is guided by location of food and the past experience of food location, in algorith $m$ this step helps to update position of best krill individual. The foraging motion of $i^{\text {th }}$ krill at its $q^{\text {th }}$ moment $\left(v_{f i}^{q}\right)$ can be expressed as $[19,46]$ :

$$
v_{f i}^{q}=0.02 \times\left[2\left(1-\frac{i \text { ter }}{i \text { ter }_{\max }}\right) f_{i} \frac{\sum_{i=1}^{N} \frac{x_{i}}{k_{i}}}{\sum_{i=1}^{N} \frac{1}{k_{i}}}+f_{i}^{\text {best }} x_{i}^{\text {best }}\right]+\omega_{x} V_{f i}^{q-1}
$$

Where $\omega_{x}$ represents the foraging motion.

\section{3) Diffusion}

The diffusion of the krill individual is considered to be a random in nature and it is incorporated to enhance the population diversity. The diffusion speed can be expressed as:

$$
v_{d i}=v_{d}^{\max } \varphi
$$

Where $v_{d}^{\max }$ is the maximum diffusion speed and $\phi$ is the random directional vector uniformly distributed between $(-1,1)$ [19].

\section{4) Position Update mechanism}

During optimization process krill regularly changes their position in search space guided by motion induced by other krill individual, foraging motion and diffusion. All motion work simultaneously and makes algorithm more powerful. Position Update mechanism of $i^{\text {th }}$ krill can be expressed as [19, 46]:

$$
x_{i}^{q+1}=x_{i}^{q}+\left(v_{i}^{q}+v_{f i}^{q}+v_{d i}^{q}\right) \times \eta \times \sum_{j=1}^{M}\left(u b_{i}-l b_{j}\right)
$$

Where $u b_{i}, l b_{j}$ the upper and lower limits of $j^{t h}$ variable, $M$ is the total number of variable and $\eta \in(0,2)$ is a constant.

The position of krill individual is updated on the bas is of their fitness, and stops as global optimal solution/ termination criteria achieved.

Also, in order to boost the exploration as well as exploitation capacity of KHA, Crossover and mutation operator as in differential evolution has been introduced here.

\section{B. KrillHerd Algorithm with Mutation and Crossover (KHAMC)}

\section{1) Crossover operator}

Krill individual position is updated on the basis of crossover probability. Updating procedure of the $j^{\text {th }}$ components of the $\mathrm{i}^{\text {th }}$ krill may be described as:

$$
\begin{gathered}
x_{i, j}=\left\{\begin{array}{rrr}
x_{r, j} & \text { if } & \text { rand } \leq C r \\
x_{i, j} & \text { if } & \text { rand }>C r
\end{array} \quad \text { where } \mathrm{r}=1,2,3 \ldots . \mathrm{N}\right. \\
C r=0.2 \times f_{i}^{\text {best }}
\end{gathered}
$$

The crossover probability decreases as fitness increases, for global best solution $\mathrm{Cr}=0$.

2) Mutation operator

$$
x_{i, j}=x_{b e s t, j}+F\left(x_{1, j}-x_{2, j}\right), F \in(0,1)
$$

With the help of mutation probability $\left(P_{m}\right)$ the 
modified value selected as:

$$
\begin{gathered}
x_{i, j}^{\text {new }}=\left\{\begin{array}{lll}
x_{r, j} & \text { if } & \text { rand } \leq P_{m} \\
x_{i, j} & \text { if } & \text { rand }>P_{m}
\end{array} \quad \text { where } \mathrm{r}=1,2,3 \ldots . \mathrm{N}\right.
\end{gathered}
$$

\section{KrillHerd Algorithm with Neighborhood Distance Concept (KHAMC)}

In order to improve the computational time, neighboring distance concept has been added here. Here distance of individual krill from their neighboring krill $\left(\right.$ dis $\left._{i, o}\right)$ can be computed as:

$$
\operatorname{dis}_{i, o}=\left\|x_{i}-x_{o}\right\|
$$

The distances so obtained are arranged in an ascending order and their respective index positions are computed. Now shorted top twenty five percent of the actual population used for computing motion induced by other krill individual and then for local swarm density calculation. This process helps to further improve the exploitation capability of KHAMC.

To analyze the performance of KHAMCD, it is tested on unimodal / multimodal benchmark functions along with two real world optimization problem of power system.

\section{PERFormance EVAluation}

All experiments were conducted on a PC with 1.80 $\mathrm{GHz}$ Intel i5 processor and $4.00 \mathrm{~GB}$ RAM. Our implementation was compiled using MATLAB. Implementation procedure of proposed algorithm for solution of problem is depicted in Fig.2. In order to examine the performance KHAMCD, it is tested on eight benchmark functions [39] as in Table 3. Further its applicability and validity is investigated using two complex constrained real world optimization problems of economic load dispatch. Comparison of results made with recent reported method to show superiority of algorithm.

\section{A. Parameter setup}

Simulation analysis were carried out with foraging speed $\left(v_{f}\right)=0.02$, the maximum induced speed $\left(v^{\max }\right)=$ 0.01 , crossover probability $(C r)=0.9$, probability of mutation $\left(P_{m}\right)=0.6$. The inertia weights $(\omega)$ are set at 0.9 at the beginning whereas 0.1 at the end and the constant $(\eta)$ set at 0.2 . The diffused speed considered as $v_{d}^{\max }=$ 0.010 which decreases linearly to 0.002 as algorithm reaches to termination criteria as maximum iteration.

\section{B. Testing of Benchmark Function}

All experiment was conducted with population size of 100 over 20 trials. Maximum iteration 100 is used for benchmark function $(f 1, f 2, f 4, f 6, f 7, f 8)$ and 300 for $(f 3)$ and 500 for ( $f 5)$.The outcome of simu lation of benchmark obtained for KHA, KHAMC and KHAMCD in terms of best fitness, worst fitness, mean fitness, standard deviation (SD) and the computational time listed in Table 4. The results are also compared with different reported results in recent literature as chaotic KHA [32, 33], fuzzy KHA [36], and KHAL [38]. Here it is observed that KHAMCD performs better in consistent manner for all problems.

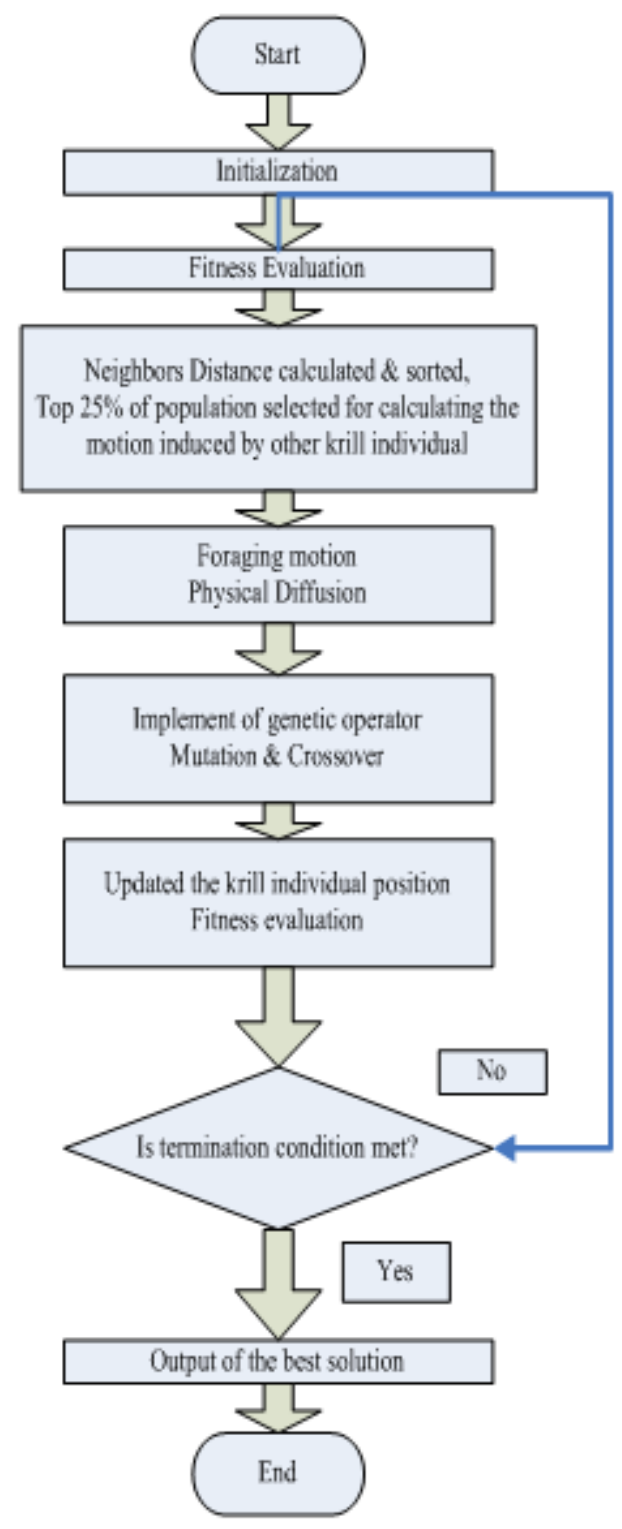

Fig.2. Flowchart of KH Algorithm with neighborhood distance concept 
Table 3. Benchmark Functions

\begin{tabular}{|c|c|c|c|c|c|}
\hline $\begin{array}{l}\text { Function } \\
\text { Name }\end{array}$ & Dimension & Type & Range & Definition & Solution \\
\hline Griewank & 30 & $\begin{array}{l}\text { Continuous, } \\
\text { differentiable, } \\
\text { non-separable, } \\
\text { scalable, } \\
\text { multimodal }\end{array}$ & {$[-100,100]$} & $f_{1}=\sum_{i=1}^{D} \frac{x_{i}^{2}}{4000}-\prod_{i=1}^{D} \cos \left(\frac{x_{i}}{\sqrt{i}}\right)+1$ & $f(x)=0$ \\
\hline Ackley & 30 & $\begin{array}{l}\text { Continuous, } \\
\text { differentiable, } \\
\text { non-separable, } \\
\text { scalable, } \\
\text { multimodal }\end{array}$ & {$[-35,35]$} & $\begin{array}{l}f_{2}=20+e-20 e \sqrt[-\frac{1}{5}]{\frac{1}{D} \sum_{i=1}^{D} x_{i}^{2}}- \\
e^{-\frac{1}{D}} \sum_{i=1}^{D} \cos \left(2 \pi x_{i}\right)\end{array}$ & $f(x)=0$ \\
\hline Booth & 30 & $\begin{array}{l}\text { Continuous, } \\
\text { differentiable, } \\
\text { non-separable, } \\
\text { non-scalable, } \\
\text { unimodal }\end{array}$ & {$[-10,10]$} & $f_{3}=\left(x_{1}+2 x_{2}-7\right)^{2}+\left(2 x_{1}+x_{2}-5\right)^{2}$ & $f(x)=0$ \\
\hline Rastrigin & 30 & $\begin{array}{l}\text { Continuous, } \\
\text { differentiable, } \\
\text { separable, } \\
\text { multimodal }\end{array}$ & {$[-5.12,5.12]$} & $f_{4}=10 D+\sum_{i=1}^{D}\left(x_{i}^{2}-10 \cos \left(2 \pi x_{i}\right)\right)$ & $f(x)=0$ \\
\hline Alpine & 30 & $\begin{array}{l}\text { Continuous, } \\
\text { Non- } \\
\text { differentiable, } \\
\text { separable, } \\
\text { scalable, } \\
\text { multimodal }\end{array}$ & {$[-10,10]$} & $f_{5}=\sum_{i=1}^{D}\left|x_{i} \sin \left(x_{i}\right)+0.1 x_{i}\right|$ & $f(x)=0$ \\
\hline Schwefel & 30 & $\begin{array}{l}\text { Continuous, } \\
\text { differentiable, } \\
\text { separable, } \\
\text { scalable, } \\
\text { multimodal }\end{array}$ & {$[-500,500]$} & $f_{6}=-\frac{1}{D} \sum_{i=1}^{D} x_{i} \sin \sqrt{\left|x_{i}\right|}$ & $f(x)=-418.983$ \\
\hline Sphere & 30 & $\begin{array}{l}\text { Continuous, } \\
\text { differentiable, } \\
\text { separable, } \\
\text { scalable, } \\
\text { multimodal }\end{array}$ & {$[-5.12,5.12]$} & $f_{7}=\sum_{i=1}^{D} x_{i}^{2}$ & $f(x)=0$ \\
\hline Rosenbrock & 30 & $\begin{array}{l}\text { Continuous, } \\
\text { differentiable, } \\
\text { non-separable, } \\
\text { scalable, } \\
\text { unimodal }\end{array}$ & {$[-2,2]$} & $f_{8}=\sum_{i=1}^{D-1}\left\lfloor 100\left(x_{i+1}-x_{i}^{2}\right)^{2}+\left(x_{i}-1\right)^{2}\right\rfloor$ & $f(x)=0$ \\
\hline
\end{tabular}

Table 4. Statistical Performance of KH variants on Benchmark Problem

\begin{tabular}{|c|c|c|c|c|c|c|c|c|c|}
\hline Function & D & Method & $\mathbf{N}$ & $\operatorname{Imax}$ & Best fitness & Worst fitness & Mean fitness & SD & $\underset{(\text { sec) }}{\text { CPU Time }}$ \\
\hline \multirow{9}{*}{$f_{1}$} & \multirow{3}{*}{2} & KHA & 100 & 100 & $1.2309 \times 10-2$ & $3.8590 \times 10-1$ & $1.2919 \times 10-1$ & $2.0732 \times 10-2$ & 10.28 \\
\hline & & KHAMC & 100 & 100 & $1.6069 \times 10-7$ & $2.4664 \times 10-3$ & $4.3616 \times 10-4$ & $1.5072 \times 10-4$ & 10.24 \\
\hline & & KHAMCD & 100 & 100 & $4.6781 \times 10-9$ & $8.7742 \times 10-4$ & $5.1675 \times 10-5$ & $4.2523 \times 10-5$ & 7.29 \\
\hline & \multirow{3}{*}{20} & KHA & 100 & 100 & $3.2079 \times 10-4$ & $2.3175 \times 10-1$ & $8.4477 \times 10-2$ & $1.7268 \times 10-2$ & 10.38 \\
\hline & & KHAMC & 100 & 100 & $1.7128 \times 10-6$ & $2.1404 \times 10-3$ & $3.1334 \times 10-4$ & $1.1908 \times 10-4$ & 10.37 \\
\hline & & KHAMCD & 100 & 100 & $5.2389 \times 10-10$ & $5.5851 \times 10-4$ & $5.0677 \times 10-5$ & $2.8277 \times 10-5$ & 7.37 \\
\hline & \multirow{3}{*}{30} & KHA & 100 & 100 & $5.6578 \times 10-4$ & $3.8556 \times 10-1$ & $5.9577 \times 10-2$ & $1.9901 \times 10-2$ & 10.43 \\
\hline & & KHAMC & 100 & 100 & $2.1187 \times 10-8$ & $7.2481 \times 10-3$ & $1.0649 \times 10-3$ & $4.2692 \times 10-4$ & 10.51 \\
\hline & & KHAMCD & 100 & 100 & $3.1535 \times 10-9$ & $1.7069 \times 10-3$ & $1.4858 \times 10-4$ & $8.9533 \times 10-5$ & 7.74 \\
\hline
\end{tabular}




\begin{tabular}{|c|c|c|c|c|c|c|c|c|c|}
\hline Function & D & Method & $\mathbf{N}$ & $\operatorname{Imax}$ & Best fitness & Worst fitness & Mean fitness & SD & $\begin{array}{c}\text { CPU Time } \\
\text { (sec) }\end{array}$ \\
\hline & & $\begin{array}{c}\text { Fuzzy KHA } \\
{[36]}\end{array}$ & 25 & 200 & $2.12 \times 10-8$ & ------- & $1.5462 \times 10-2$ & ------- & ------- \\
\hline & & $\begin{array}{c}\text { Choatic } \\
\text { KHA [32] }\end{array}$ & ------ & 500 & ------- & ------- & $5.6939 \times 10-2$ & $2.6886 \times 10-2$ & ------- \\
\hline & & $\begin{array}{c}\text { Choatic } \\
\text { KHA [33] }\end{array}$ & 25 & 200 & $1.32 \times 10-7$ & ------- & $1.44 \times 10-2$ & ------- & ------- \\
\hline & & KHAL [38] & 10 & 10000 & ------ & ------- & $4.1 \times 10-3$ & $6.8 \times 10-3$ & ------ \\
\hline \multirow{12}{*}{$f_{2}$} & \multirow{3}{*}{2} & KHA & 100 & 100 & 2.7450 & $1.2069 \times 10+1$ & 7.5091 & $6.4341 \times 10-1$ & 10.48 \\
\hline & & KHAMC & 100 & 100 & $1.8975 \times 10-3$ & $1.6410 \times 10-1$ & $4.9798 \times 10-2$ & $9.3817 \times 10-3$ & 10.33 \\
\hline & & KHAMCD & 100 & 100 & $2.7828 \times 10-5$ & $2.0834 \times 10-2$ & $4.0406 \times 10-3$ & $1.4270 \times 10-3$ & 7.33 \\
\hline & \multirow{3}{*}{20} & KHA & 100 & 100 & 2.2341 & $1.2621 \times 10+1$ & 6.7996 & $6.0828 \times 10-1$ & 10.39 \\
\hline & & KHAMC & 100 & 100 & $2.9504 \times 10-2$ & $1.7021 \times 10-1$ & $7.1720 \times 10-2$ & $8.0913 \times 10-3$ & 10.44 \\
\hline & & KHAMCD & 100 & 100 & $1.2246 \times 10-4$ & $2.3592 \times 10-2$ & $3.2265 \times 10-3$ & $1.3799 \times 10-3$ & 7.23 \\
\hline & \multirow{6}{*}{30} & KHA & 100 & 100 & 1.5591 & $1.5017 \times 10+1$ & 7.4434 & $8.0605 \times 10-1$ & 10.35 \\
\hline & & KHAMC & 100 & 100 & $5.8713 \times 10-3$ & $3.2497 \times 10-1$ & $6.8098 \times 10-2$ & $1.5643 \times 10-2$ & 10.43 \\
\hline & & KHAMCD & 100 & 100 & $1.0746 \times 10-5$ & $4.8179 \times 10-2$ & $6.7143 \times 10-3$ & $2.4956 \times 10-3$ & 7.35 \\
\hline & & $\begin{array}{c}\text { Fuzzy KHA } \\
{[36]}\end{array}$ & 25 & 200 & $1.119 \times 10-4$ & ------ & $3.3439 \times 10-1$ & ------ & ------ \\
\hline & & $\begin{array}{c}\text { Choatic } \\
\text { KHA [33] }\end{array}$ & 25 & 200 & $1.268 \times 10-4$ & ------- & $1.8594 \times 10-1$ & ------- & ------- \\
\hline & & KHAL [38] & 10 & 10000 & ------ & ------ & $6.523 \times 10-1$ & $2.323 \times 10-1$ & ------- \\
\hline \multirow{6}{*}{$f_{3}$} & \multirow{3}{*}{2} & KHA & 200 & 300 & $2.2981 \times 10-3$ & $2.8530 \times 10-1$ & $6.0273 \times 10-2$ & $1.4454 \times 10-2$ & 120.56 \\
\hline & & KHAMC & 200 & 300 & $9.7826 \times 10-6$ & $8.6048 \times 10-1$ & $7.7090 \times 10-2$ & $4.7057 \times 10-2$ & 120.81 \\
\hline & & KHAMCD & 200 & 300 & $4.2118 \times 10-5$ & $4.5324 \times 10-1$ & $4.9316 \times 10-2$ & $2.3785 \times 10-2$ & 105.88 \\
\hline & \multirow{3}{*}{20} & KHA & 100 & 300 & $3.6026 \times 10-4$ & $3.1339 \times 10-1$ & $9.8358 \times 10-2$ & $2.3722 \times 10-2$ & 30.48 \\
\hline & & KHAMC & 100 & 300 & $1.9298 \times 10-5$ & $6.5852 \times 10-1$ & $7.5571 \times 10-2$ & $3.5505 \times 10-2$ & 30.83 \\
\hline & & KHAMCD & 100 & 300 & $8.4031 \times 10-6$ & $9.3225 \times 10-1$ & $6.4951 \times 10-2$ & $4.5088 \times 10-2$ & 21.77 \\
\hline \multirow{13}{*}{$f_{4}$} & \multirow{3}{*}{2} & KHA & 100 & 100 & $3.3561 \times 10-5$ & $6.4810 \times 10-1$ & $8.4018 \times 10-2$ & $3.4771 \times 10-2$ & 10.23 \\
\hline & & KHAMC & 100 & 100 & $3.6062 \times 10-6$ & $1.0730 \times 10-2$ & $1.7521 \times 10-3$ & $5.1410 \times 10-4$ & 10.06 \\
\hline & & KHAMCD & 100 & 100 & $3.2963 \times 10-7$ & $1.7130 \times 10-3$ & $3.2657 \times 10-4$ & $9.5710 \times 10-5$ & 7.19 \\
\hline & \multirow{3}{*}{20} & KHA & 100 & 100 & $1.3061 \times 10-3$ & $6.2422 \times 10-1$ & $6.8567 \times 10-2$ & $2.9756 \times 10-2$ & 10.21 \\
\hline & & KHAMC & 100 & 100 & $7.3550 \times 10-6$ & $3.5432 \times 10-2$ & $8.3993 \times 10-3$ & $1.9862 \times 10-3$ & 10.19 \\
\hline & & KHAMCD & 100 & 100 & $1.4927 \times 10-7$ & $1.6322 \times 10-3$ & $2.6607 \times 10-4$ & $8.5491 \times 10-5$ & 7.49 \\
\hline & \multirow{7}{*}{30} & KHA & 100 & 100 & $1.1504 \times 10-5$ & $7.2139 \times 10-1$ & $9.1691 \times 10-2$ & $3.9918 \times 10-2$ & 10.45 \\
\hline & & KHAMC & 100 & 100 & $1.4198 \times 10-4$ & $8.1789 \times 10-2$ & $1.3374 \times 10-2$ & $4.9771 \times 10-3$ & 10.41 \\
\hline & & KHAMCD & 100 & 100 & $1.4273 \times 10-5$ & $4.1291 \times 10-3$ & $5.1064 \times 10-4$ & $2.3012 \times 10-4$ & 7.63 \\
\hline & & $\begin{array}{c}\text { Fuzzy KHA } \\
{[36]}\end{array}$ & 25 & 200 & 1.025096 & ------- & 3.980158 & ------- & ------ \\
\hline & & $\begin{array}{c}\text { Choatic } \\
\text { KHA [32] }\end{array}$ & ------ & 500 & ------- & ------- & 20.50905 & 4.3942 & ------- \\
\hline & & $\begin{array}{c}\text { Choatic } \\
\text { KHA [33] }\end{array}$ & 25 & 200 & $3.072 \times 10-2$ & ------- & $2.6523 \times 10-1$ & ------- & ------- \\
\hline & & KHAL [38] & 10 & 10000 & ------- & ------- & 9.6714 & 6.5899 & ------- \\
\hline \multirow{6}{*}{$f_{5}$} & \multirow{3}{*}{2} & KHA & 100 & 500 & $5.4811 \times 10-4$ & $1.4929 \times 10-1$ & $3.3880 \times 10-2$ & $7.5166 \times 10-3$ & 50.56 \\
\hline & & KHAMC & 100 & 500 & $5.2634 \times 10-6$ & $4.9520 \times 10-4$ & $9.1777 \times 10-5$ & $3.0892 \times 10-5$ & 51.08 \\
\hline & & KHAMCD & 100 & 500 & $2.2140 \times 10-9$ & $9.5300 \times 10-8$ & $2.8705 \times 10-8$ & $4.7439 \times 10-9$ & 35.57 \\
\hline & \multirow{3}{*}{20} & KHA & 100 & 500 & $1.8992 \times 10-3$ & 1.8324 & $1.2769 \times 10-1$ & $8.7695 \times 10-2$ & 51.33 \\
\hline & & KHAMC & 100 & 500 & $5.8783 \times 10-1$ & 26.097 & 5.5406 & 1.2903 & 51.30 \\
\hline & & KHAMCD & 100 & 500 & $1.6978 \times 10-4$ & 12.678 & 2.9513 & $8.6842 \times 10-1$ & 35.83 \\
\hline \multirow{10}{*}{$f_{6}$} & \multirow{3}{*}{2} & KHA & 100 & 100 & -726.68 & -597.23 & -661.66 & 8.353 & 10.20 \\
\hline & & KHAMC & 100 & 100 & -837.97 & -716.75 & -824.78 & 6.152 & 10.28 \\
\hline & & KHAMCD & 100 & 100 & -837.92 & -717.73 & -814.68 & 9.239 & 7.39 \\
\hline & \multirow{3}{*}{20} & KHA & 100 & 100 & -811.76 & -546.68 & -686.55 & 17.946 & 10.94 \\
\hline & & KHAMC & 100 & 100 & -837.96 & -715.08 & -815.69 & 9.6001 & 10.70 \\
\hline & & KHAMCD & 100 & 100 & -837.94 & -599.54 & -812.52 & 13.560 & 7.75 \\
\hline & \multirow{4}{*}{30} & KHA & 100 & 100 & -828.97 & -583.27 & -709.08 & 18.935 & 11.57 \\
\hline & & KHAMC & 100 & 100 & -837.92 & -712.30 & -799.04 & 12.022 & 12.66 \\
\hline & & KHAMCD & 100 & 100 & -837.91 & -703.71 & -809.94 & 10.752 & 7.53 \\
\hline & & $\begin{array}{c}\text { Fuzzy KHA } \\
{[36]}\end{array}$ & 25 & 200 & $6.38 \times 10-4$ & ------ & $5.241 \times 10-3$ & ------ & ------- \\
\hline
\end{tabular}




\begin{tabular}{|c|c|c|c|c|c|c|c|c|c|}
\hline Function & D & Method & $\mathbf{N}$ & $\operatorname{Imax}$ & Best fitness & Worst fitness & Mean fitness & SD & $\begin{array}{c}\text { CPU Time } \\
\text { (sec) }\end{array}$ \\
\hline & & $\begin{array}{c}\text { Choatic } \\
\text { KHA [32] }\end{array}$ & ------ & 500 & ------- & ------- & -5905.55 & 2361.01 & ------- \\
\hline & & $\begin{array}{c}\text { Choatic } \\
\text { KHA [33] }\end{array}$ & 25 & 200 & $1.874 \times 10-4$ & ------- & $2.2151 \times 10-2$ & ------- & ------- \\
\hline \multirow{13}{*}{$f_{7}$} & \multirow{3}{*}{2} & KHA & 100 & 100 & $9.3244 \times 10-5$ & $1.3229 \times 10-1$ & $1.8492 \times 10-2$ & $7.1446 \times 10-3$ & 11.72 \\
\hline & & KHAMC & 100 & 100 & $6.2953 \times 10-8$ & $2.2142 \times 10-5$ & $6.6838 \times 10-6$ & $1.7277 \times 10-6$ & 11.97 \\
\hline & & KHAMCD & 100 & 100 & $1.0547 \times 10-10$ & $2.2662 \times 10-5$ & $2.4920 \times 10-6$ & $1.4490 \times 10-6$ & 8.88 \\
\hline & \multirow{3}{*}{20} & KHA & 100 & 100 & $8.0312 \times 10-5$ & $2.3803 \times 10-2$ & $5.2903 \times 10-3$ & $1.5889 \times 10-3$ & 12.00 \\
\hline & & KHAMC & 100 & 100 & $1.3340 \times 10-7$ & $4.9025 \times 10-5$ & $1.0330 \times 10-5$ & $3.1152 \times 10-6$ & 11.76 \\
\hline & & KHAMCD & 100 & 100 & $2.9633 \times 10-10$ & $1.8687 \times 10-5$ & $2.7952 \times 10-6$ & $1.0101 \times 10-6$ & 8.28 \\
\hline & \multirow{7}{*}{30} & KHA & 100 & 100 & $2.8513 \times 10-5$ & $8.8107 \times 10-2$ & $9.8531 \times 10-3$ & $4.4336 \times 10-3$ & 11.49 \\
\hline & & KHAMC & 100 & 100 & $2.8175 \times 10-7$ & $7.2317 \times 10-5$ & $1.4779 \times 10-5$ & $4.9660 \times 10-6$ & 11.37 \\
\hline & & KHAMCD & 100 & 100 & $6.5458 \times 10-12$ & $7.4766 \times 10-6$ & $1.3395 \times 10-6$ & $4.1764 \times 10-7$ & 7.96 \\
\hline & & $\begin{array}{c}\text { Fuzzy KHA } \\
{[36]}\end{array}$ & 25 & 200 & $4.68 \times 10-5$ & ------- & 0.000258 & ------- & ------- \\
\hline & & $\begin{array}{c}\text { Choatic } \\
\text { KHA [32] }\end{array}$ & ------ & 500 & ------ & ------ & 0.3541 & 0.318221 & ------ \\
\hline & & $\begin{array}{c}\text { Choatic } \\
\text { KHA [33] }\end{array}$ & 25 & 200 & 0.0195 & ------ & 0.24187 & ------ & ------ \\
\hline & & KHAL [38] & 10 & 10000 & ------ & ------- & 0.2504 & 0.2135 & ------- \\
\hline \multirow{12}{*}{$f_{8}$} & \multirow{3}{*}{2} & KHA & 100 & 100 & $5.3259 \times 10-6$ & $5.5072 \times 10-2$ & $5.5912 \times 10-3$ & $2.7019 \times 10-3$ & 11.77 \\
\hline & & KHAMC & 100 & 100 & $1.2777 \times 10-2$ & $6.3791 \times 10-1$ & $1.5062 \times 10-1$ & $3.0501 \times 10-2$ & 11.72 \\
\hline & & KHAMCD & 100 & 100 & $1.5709 \times 10-2$ & $5.9110 \times 10-1$ & $1.8952 \times 10-1$ & $3.0650 \times 10-2$ & 8.17 \\
\hline & \multirow{3}{*}{20} & KHA & 100 & 100 & $1.2544 \times 10-4$ & $9.0696 \times 10-2$ & $1.8495 \times 10-2$ & $5.44680 \times 10-3$ & 11.85 \\
\hline & & KHAMC & 100 & 100 & $6.2359 \times 10-3$ & $3.2100 \times 10-1$ & $9.6901 \times 10-2$ & $1.7849 \times 10-2$ & 11.94 \\
\hline & & KHAMCD & 100 & 100 & $3.7283 \times 10-3$ & $5.8347 \times 10-1$ & $1.1992 \times 10-1$ & $2.8997 \times 10-2$ & 8.71 \\
\hline & \multirow{6}{*}{30} & KHA & 100 & 100 & $2.7725 \times 10-5$ & $1.9508 \times 10-1$ & $4.2240 \times 10-2$ & $1.19939 \times 10-2$ & 11.76 \\
\hline & & KHAMC & 100 & 100 & $4.8975 \times 10-3$ & $2.8506 \times 10-1$ & $1.2771 \times 10-1$ & $1.7381 \times 10-2$ & 11.61 \\
\hline & & KHAMCD & 100 & 100 & $3.9030 \times 10-3$ & $2.3346 \times 10-1$ & $1.1508 \times 10-1$ & $1.8295 \times 10-2$ & 8.26 \\
\hline & & $\begin{array}{c}\text { Fuzzy KHA } \\
{[36]} \\
\end{array}$ & 25 & 200 & 3.18330701 & ------- & 7.807 & ------- & ------- \\
\hline & & $\begin{array}{c}\text { Choatic } \\
\text { KHA [33] }\end{array}$ & 25 & 200 & $3.983 \times 10-5$ & ------- & 0.000165 & ------- & ------- \\
\hline & & KHAL [38] & 10 & 10000 & ------- & ------- & 0.0048 & 0.0108 & ------- \\
\hline
\end{tabular}

D: Dimension, SD: Standard Deviation
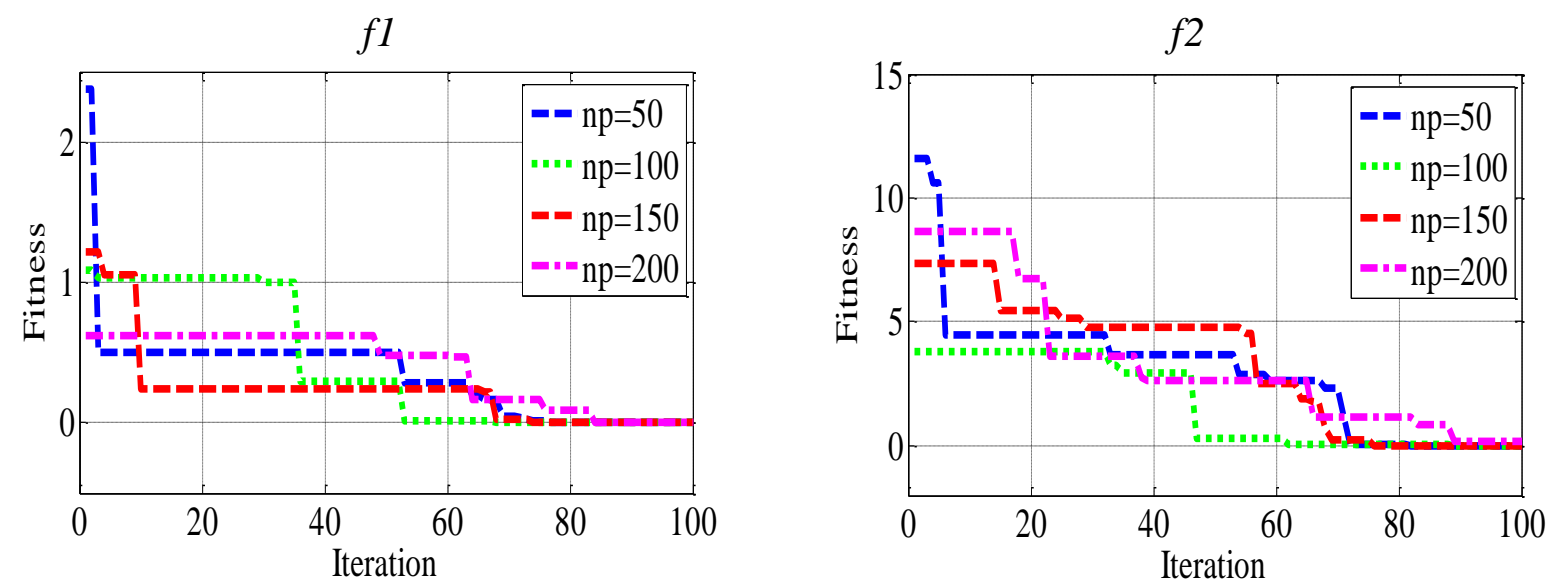


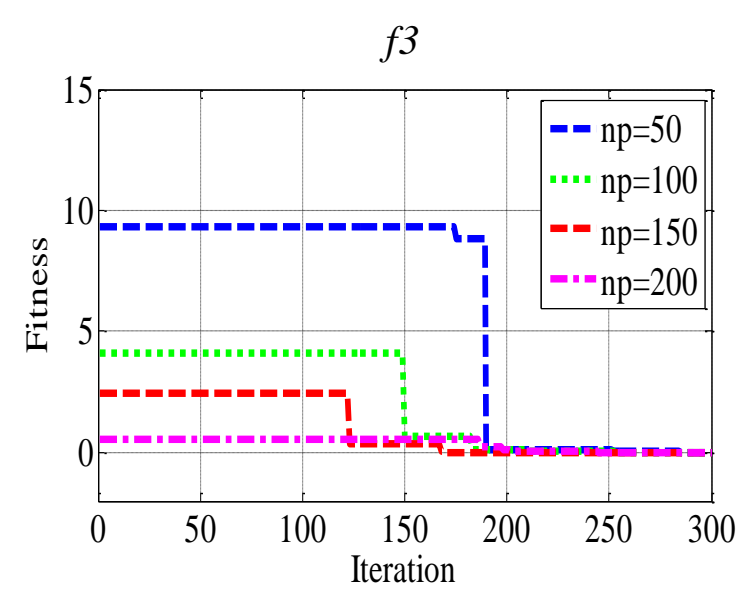

f5

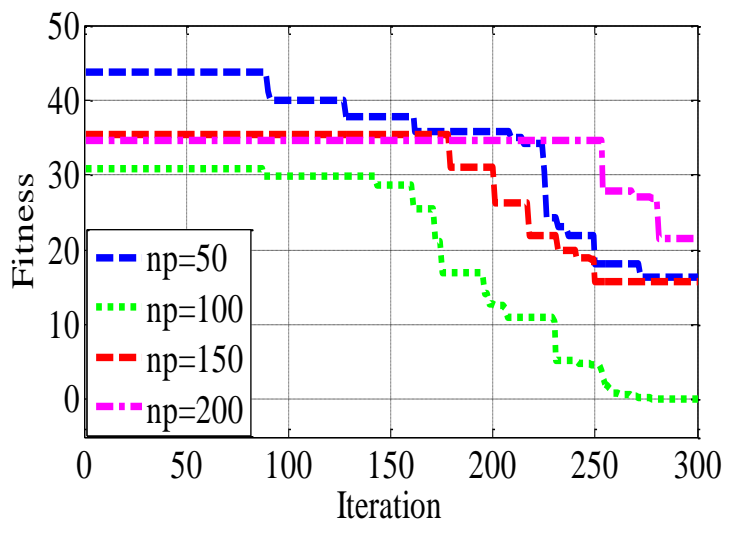

$f 7$

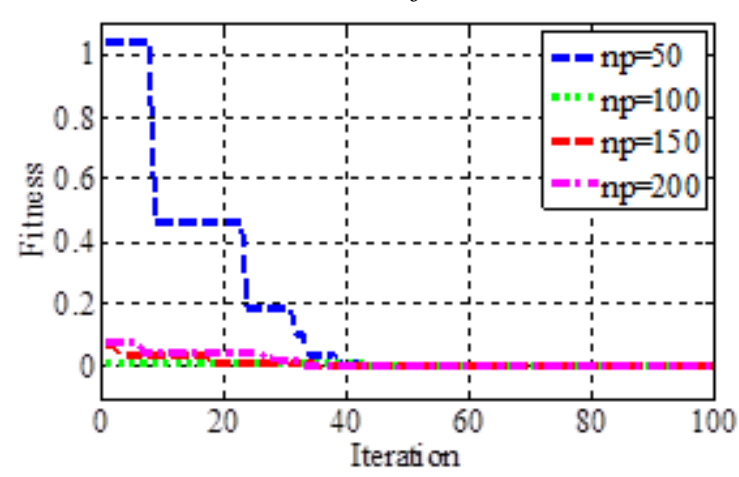

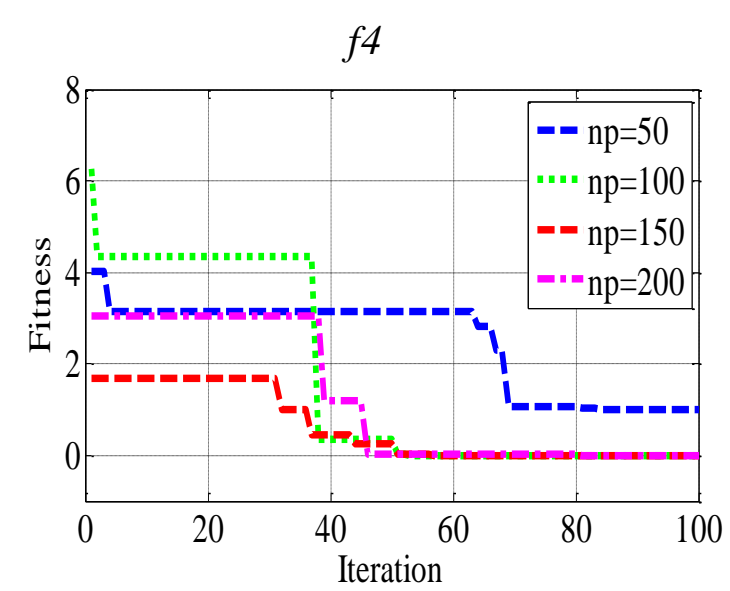
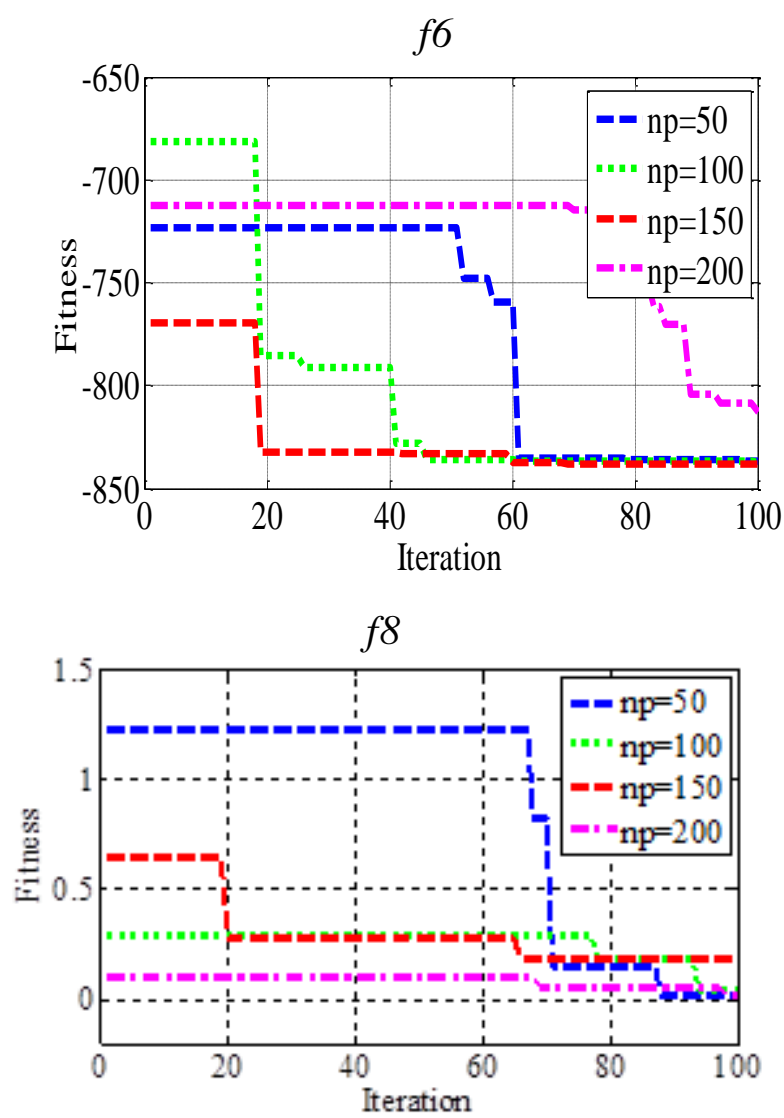

Fig.3. Convergence Characteristic of KHAMCD for different population on 20-D benchmark

\section{Comparison of convergence characteristics}

Convergence characteris tic of KHAMCD algorith $m$ are plotted with change in population in Fig 3 which shows that with increase in population the convergence improves. To make fair comparison between KHA, KHAMC and KHAMCD the convergence characteristics for different benchmark functions are compared in Fig 4, and the performance of KHAMCD is found to be better. 

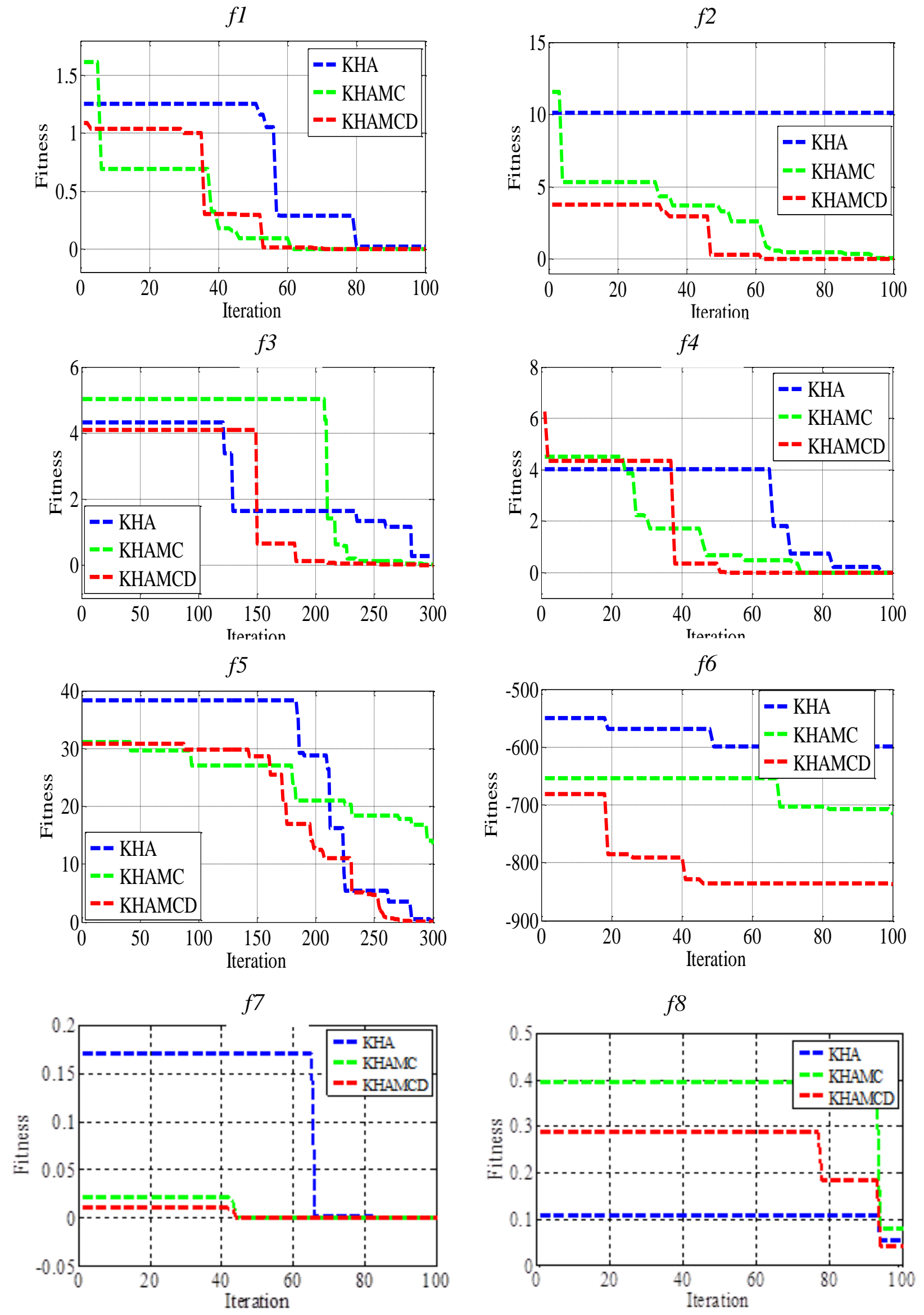

Fig.4. Comparison of convergence characteristics of variants of KHA 


\section{Consistency Analysis}

As proposed KHAMCD uses random operators similar to other stochastic search optimization technique and hence in every trial the algorithm converge to slightly different value. Therefore, it is general practice to conduct various trials and statistical analysis is also carried out on benchmark functions as shown in Table 5. Also it is illustrated using Fig 5 with different population on 20-D. It is observed that population size of 100 good consistencies compared to other population for $f 1-f 5, f 8$ whereas $f 6, f 7$ have better consistency with population size of 150 and 200.

Table 5. Consistency Analysis of KHAMCD with different population size

\begin{tabular}{|c|c|c|c|c|c|c|}
\hline $\begin{array}{c}\text { Function } \\
\text { Name }\end{array}$ & $N$ & $\operatorname{Imax}$ & Best fitness & Worst fitness & Mean & $\begin{array}{l}\text { Standard } \\
\text { Deviation }\end{array}$ \\
\hline \multirow{4}{*}{$f 1$} & 50 & 100 & $5.2736 \times 10-14$ & $1.4562 \times 10-1$ & $2.1843 \times 10-2$ & $1.1627 \times 10-2$ \\
\hline & 100 & 100 & $8.6808 \times 10-13$ & $3.9258 \times 10-4$ & $2.4350 \times 10-5$ & $1.9234 \times 10-2$ \\
\hline & 150 & 100 & $1.4977 \times 10-13$ & $1.5364 \times 10-4$ & $1.0477 \times 10-5$ & $7.6011 \times 10-6$ \\
\hline & 200 & 100 & $1.7531 \times 10-10$ & $1.0739 \times 10-4$ & $8.9068 \times 10-6$ & $5.3135 \times 10-6$ \\
\hline \multirow{4}{*}{$f 2$} & 50 & 100 & $1.2343 \times 10-6$ & $4.0871 \times 10-2$ & $3.2304 \times 10-3$ & $2.2343 \times 10-3$ \\
\hline & 100 & 100 & $7.2152 \times 10-7$ & $4.5232 \times 10-2$ & $4.8895 \times 10-3$ & $2.4332 \times 10-3$ \\
\hline & 150 & 100 & $4.8540 \times 10-6$ & $4.7975 \times 10-2$ & $5.8461 \times 10-3$ & $2.4157 \times 10-3$ \\
\hline & 200 & 100 & $3.2882 \times 10-6$ & $3.2535 \times 10-2$ & $1.0839 \times 10-2$ & $2.4090 \times 10-3$ \\
\hline \multirow{4}{*}{$f 3$} & 50 & 300 & $7.8347 \times 10-5$ & $9.6875 \times 10-1$ & $7.5608 \times 10-2$ & $4.7113 \times 10-2$ \\
\hline & 100 & 300 & $3.1727 \times 10-4$ & $1.8439 \times 10-1$ & $2.4747 \times 10-2$ & $1.0687 \times 10-2$ \\
\hline & 150 & 300 & $1.6369 \times 10-4$ & $7.3522 \times 10-1$ & $7.0285 \times 10-2$ & $3.9045 \times 10-2$ \\
\hline & 200 & 300 & $1.1243 \times 10-4$ & $3.1155 \times 10-1$ & $2.9850 \times 10-2$ & $1.5157 \times 10-2$ \\
\hline \multirow{4}{*}{$f 4$} & 50 & 100 & $1.2438 \times 10-8$ & $9.9565 \times 10-1$ & $8.0639 \times 10-2$ & $5.3946 \times 10-2$ \\
\hline & 100 & 100 & $2.3078 \times 10-7$ & $1.8638 \times 10-3$ & $4.6818 \times 10-4$ & $1.0556 \times 10-4$ \\
\hline & 150 & 100 & $7.4842 \times 10-7$ & $1.6456 \times 10-3$ & $2.8183 \times 10-4$ & $7.2329 \times 10-5$ \\
\hline & 200 & 100 & $1.8448 \times 10-5$ & $1.4651 \times 10-3$ & $2.8534 \times 10-4$ & $6.6840 \times 10-5$ \\
\hline \multirow{4}{*}{$f 5$} & 50 & 500 & $2.5094 \times 10-2$ & 20.524 & 2.9311 & 1.0796 \\
\hline & 100 & 500 & $3.3115 \times 10-3$ & 31.275 & 6.3757 & 1.8344 \\
\hline & 150 & 500 & $1.3246 \times 10-2$ & 20.726 & 8.3712 & 1.7670 \\
\hline & 200 & 500 & $2.9093 \times 10-3$ & 25.036 & 8.4455 & 1.9047 \\
\hline \multirow{4}{*}{ f6 } & 50 & 100 & -837.94 & -685.33 & -780.43 & 13.311 \\
\hline & 100 & 100 & -837.92 & -663.17 & -789.62 & 13.822 \\
\hline & 150 & 100 & -837.96 & -719.13 & -824.79 & 7.8827 \\
\hline & 200 & 100 & -837.93 & -717.05 & -818.89 & 9.4561 \\
\hline \multirow{4}{*}{$f 7$} & 50 & 100 & $7.1146 \times 10-11$ & $5.8820 \times 10-5$ & $3.6337 \times 10-6$ & $2.8627 \times 10-6$ \\
\hline & 100 & 100 & $1.0717 \times 10-10$ & $4.2097 \times 10-5$ & $3.2639 \times 10-6$ & $2.0344 \times 10-6$ \\
\hline & 150 & 100 & $1.6174 \times 10-08$ & $1.4042 \times 10-5$ & $3.0890 \times 10-6$ & $9.3391 \times 10-7$ \\
\hline & 200 & 100 & $1.6004 \times 10-12$ & $5.7270 \times 10-6$ & $1.3783 \times 10-6$ & $3.8511 \times 10-7$ \\
\hline \multirow{4}{*}{$f 8$} & 50 & 100 & $1.9340 \times 10-2$ & 1.1057 & 0.27688 & 0.057311 \\
\hline & 100 & 100 & $3.7283 \times 10-3$ & 0.58347 & 0.11992 & 0.028997 \\
\hline & 150 & 100 & $4.1531 \times 10-3$ & 0.24786 & 0.097353 & 0.016987 \\
\hline & 200 & 100 & $9.8037 \times 10-3$ & 0.19688 & 0.085857 & 0.015160 \\
\hline
\end{tabular}



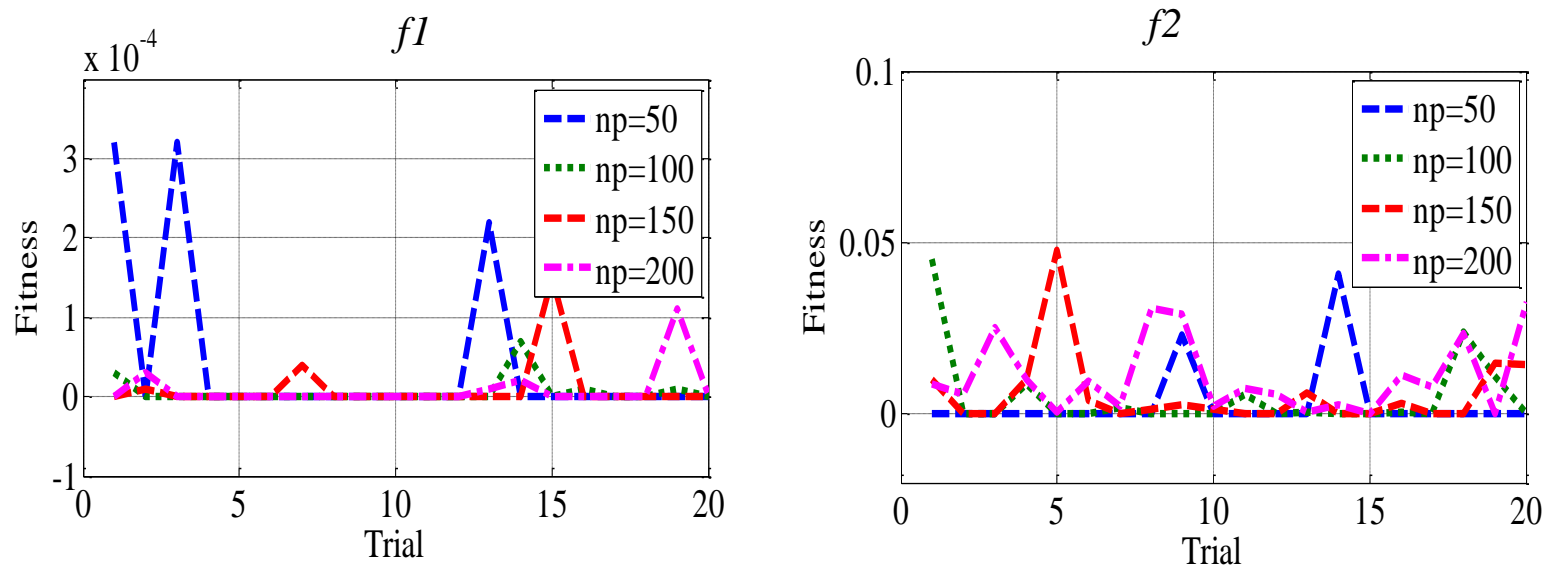

$f 3$

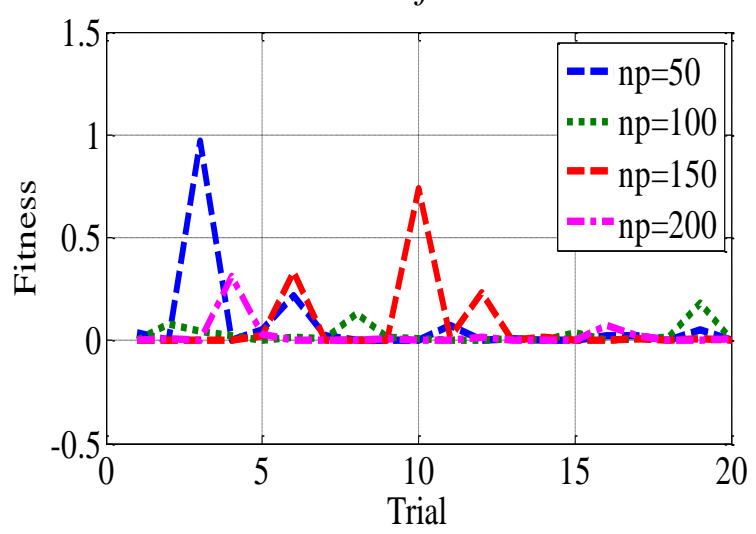

f4

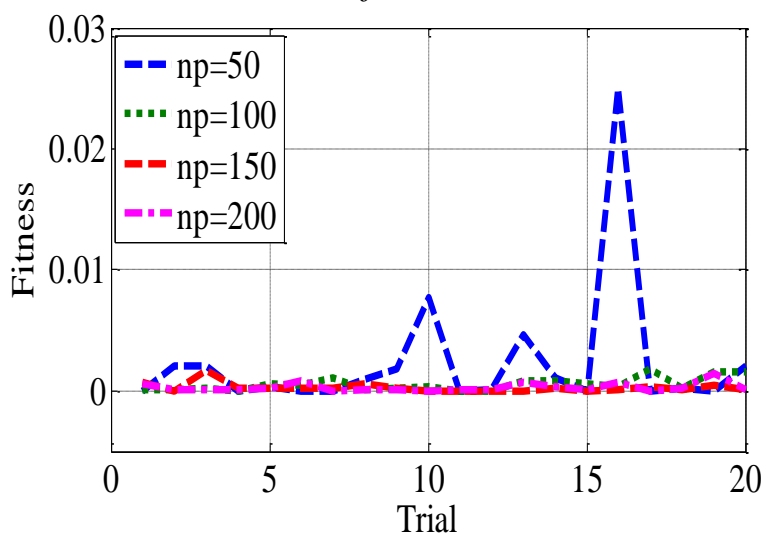

f5
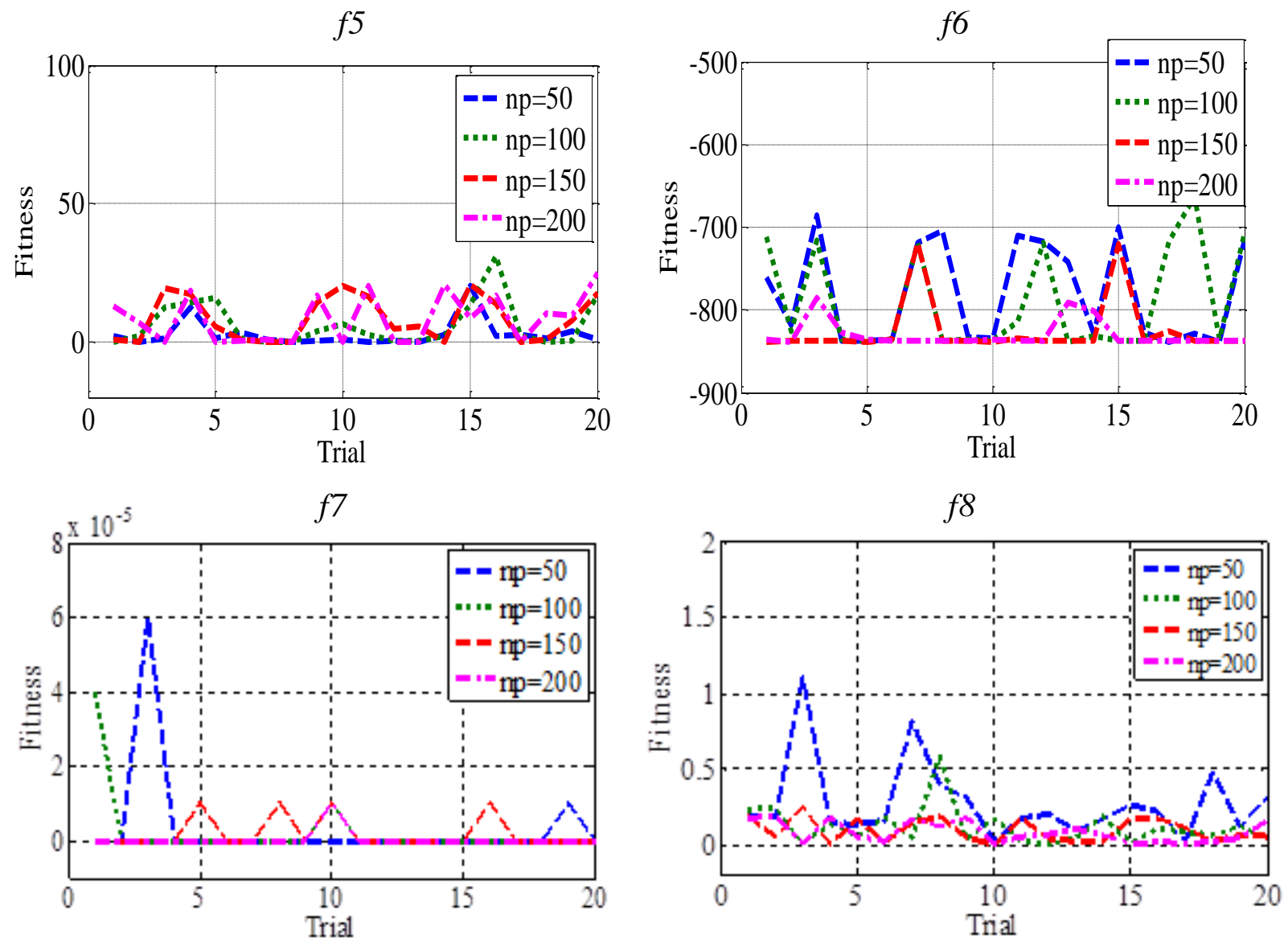

Fig.5. Consistency Analysis of KHAMCD on 20-D benchmark 


\section{ECONOMIC LOAD DISPATCH PROBLEM}

In this section KHAMCD algorith $m$ is used to optimize practical Economic Load Dispatch (ELD) problem. ELD is key issue related with power system operation and control with goal is to find out most reliable, efficient and low cost operation of power system that can capable to match required power demand by proper dispatch of output from committed generators. ELD is complex optimization problem with main objective is to minimize the cost function and has to satisfy the operating constraints too. These types of problem have many minima and hence classical method unable to provide global best solution. On the other hand population based stochastic search NI method often provides near global solution is a better choice for solution of these types of problem.

Various optimization techniques as lamda iteration [41], quadratic programming and GAMS [52], differential evolution(DE)[43], teaching learning based optimization(TLBO)[44], Chemical Reaction Optimization (CRO) [45], krill herd algorithm(KHA) [46,53] invasive weed optimization(IW O) [47], gravitational search (GSA)[48], flower pollination algorithm(FPA) [49], Artificial Bee Colony(ABC) [50], rooted tree optimization(RTO) [51] and hybrid DE with particle swarm optimization (PSO) [52] are successfully applied for solution of ELD problem.A comprehensive review of NI techniques for solution ELD problem is presented in [40]

To validate the performance of proposed KHAMCD it is tested on two standards complex constraint comparative medium and large scale ELD problem as below.

\section{A. ELD formulation as cost function}

The objective function corresponding to the power generation cost can be approximated as quadratic function of the active power outputs of committed the generating units. Symbolically, it is represented as:

$$
\begin{aligned}
& \text { Minimize } F_{T}=\sum_{i=1}^{N} f_{i}\left(P_{i}\right) \\
& \text { Where } f_{i}\left(P_{i}\right)=a_{i} P_{i}^{2}+b_{i} P_{i}+C_{i} \\
& \mathrm{i}=1,2,3, \ldots \ldots \ldots . \mathrm{N}
\end{aligned}
$$

$a_{i}, b_{i}$ and $c_{i}$ represents its cost coefficients. Expression for cost function (14) corresponding to $i^{\text {th }}$ generating unit, $\mathrm{P}_{\mathrm{i}}$ is the real power output (MW) and $N$ is the number of online generator used for power dispatched. The cost function with valve point loading effect is computed as [48,49:

$$
f_{i}\left(P_{i}\right)=a_{i} P_{i}^{2}+b_{i} P_{i}+c_{i}+\left|e_{i} \times \sin \left\{f_{i} \times\left(P_{i}^{\min }-P_{i}\right)\right\}\right|
$$

Where $e_{i}$ and $f_{i}$ indicating the valve point effect of $i^{\text {th }}$ generator.
Subjected to constrains as

\section{1) Power balance constraint}

$$
\sum_{i=1}^{N} P_{i}=P_{D}+P_{L}
$$

The transmission losses occurring in the system can be expressed using B-loss coefficients as [41]:

$$
P_{L}=\sum_{i=1}^{N} P_{i} B_{i j} P_{j}+\sum_{i=1}^{N} P_{i} B_{i 0}+B_{00}
$$

\section{2) Operating limit constraint}

$$
P_{i}^{\min } \leq P_{i} \leq P_{i}^{\min }, \quad \text { where } \mathrm{i}=1,2, \ldots . \mathrm{N}
$$

The generated output of $i^{\text {th }}$ generator should lie between specified lower and upper limit.

\section{3) Constraints due to prohibited operating zones}

The prohibited operating zones (POZ) are the certain range of output power of a generator, in that rage of operation unnecessary vibration in turbine shaft takes place which may damage the shaft and bearing and hence operation is avoided in such regions. POZ makes the objective function discontinuous, therefore feasible operating zones of power generating unit can be depicted as:

$$
\left\{\begin{array}{c}
P_{i}^{\min } \leq P_{i}^{t} \leq \underline{P}_{i}^{1} \\
P_{i}^{-x-1} \leq P_{i}^{t} \leq \underline{P}_{i}^{x} \\
P_{G i}^{-n z} \leq P_{G i} \leq P_{G i}^{\max }
\end{array} \quad x=2,3 \ldots \ldots . n z,\right\}
$$

Where, $\underline{P}_{i}^{x}$ and $\overline{\boldsymbol{P}}_{i}^{x}$ are the lower and upper operating limits of the $x^{\text {th }}$ prohibited operation zone for $i^{t h}$ power generating unit.

\section{B. Results and discussion}

The applicability and viability of the aforementioned proposed KHAMCD algorithm for practical applications has been tested on two different test cases of ELD problem with population size $(\mathrm{N})$ set at 100 and other parameter are similar as in section III.A.

\section{1) Fifteen unit system with POZ and loss}

The system contains fifteen thermal generating units. Four power generating unit as 2, 5, 6 and 12 has prohibited operating zones. The fuel cost coefficient data and transmission line loss coefficient are adopted as per [46]. The total load demand on the system is $2630 \mathrm{MW}$. The optimum generation schedule obtained by proposed algorithm is presented in Table 6 and the statistical comparis on of result is made in Table 7 respectively. The optimum generation cost obtained by KHAMCD $32548.0031 \$ / \mathrm{hr}$ which is found to be slightly inferior to KH IV [46], but considering overall statis tical evaluation, the performance of KHAMCD is found to be better and 
consistent. The convergence characteristic for this test case is plotted in Fig.6.

It can be seen from results that the KHAMCD technique provides better results while satisfying the as sociated operating constraints.

Table 6. Optimum generation of KHAMCD for Test Case 1

\begin{tabular}{|c|c|c|c|c|c|}
\hline Unit & KH IV [46 ] & KHAMCD & Unit & KH IV [46 ] & KHAMCD \\
\hline$P 1$ & 455 & 455 & $P 10$ & 31.2698 & 35.6215 \\
\hline$P 2$ & 455 & 455 & $P 11$ & 76.7013 & 74.3593 \\
\hline$P 3$ & 130 & 130 & $P 12$ & 80 & 80 \\
\hline$P 4$ & 130 & 130 & $P 13$ & 25 & 25 \\
\hline$P 5$ & 233.8017 & 231.7891 & $P 14$ & 15 & 15 \\
\hline$P 6$ & 460 & 460 & $P 15$ & 15 & 15 \\
\hline$P 7$ & 465 & 465 & $\sum P_{i}(\mathrm{MW})$ & 2656.7728 & 2656.7699 \\
\hline$P 8$ & 60 & 60 & Ploss $(\mathrm{MW})$ & 26.7673 & 26.7699 \\
\hline$P 9$ & 25 & 25 & Total cost $(\$ / \mathrm{h})$ & 32547.3700 & 32548.0031 \\
\hline
\end{tabular}

Table 7. Statistical results of KHAMCD for Test Case 1

\begin{tabular}{|c|c|c|c|c|c|}
\hline Method & Min Cost $(\$ \mathbf{h r})$ & Mean $\operatorname{Cost}(\mathbf{\$} \mathbf{h r})$ & Max $\operatorname{Cost} \mathbf{( \$ / h r )}$ & S.D & CPU time $(\mathbf{s e c})$ \\
\hline KH IV[46 ] & 32547.3700 & 32548.1348 & 32548.9326 & NA & NA \\
\hline DEPSO[54 ] & 32588.8100 & 32588.9900 & 32591.4900 & 4.0200 & 1.9600 \\
\hline DPD [54] & 32548.5857 & 32556.6793 & 32564.4051 & 2.0956 & 1.9800 \\
\hline KHAMCD & 32548.0031 & 32548.1020 & 32548.354 & 0.1570 & 1.870 \\
\hline
\end{tabular}

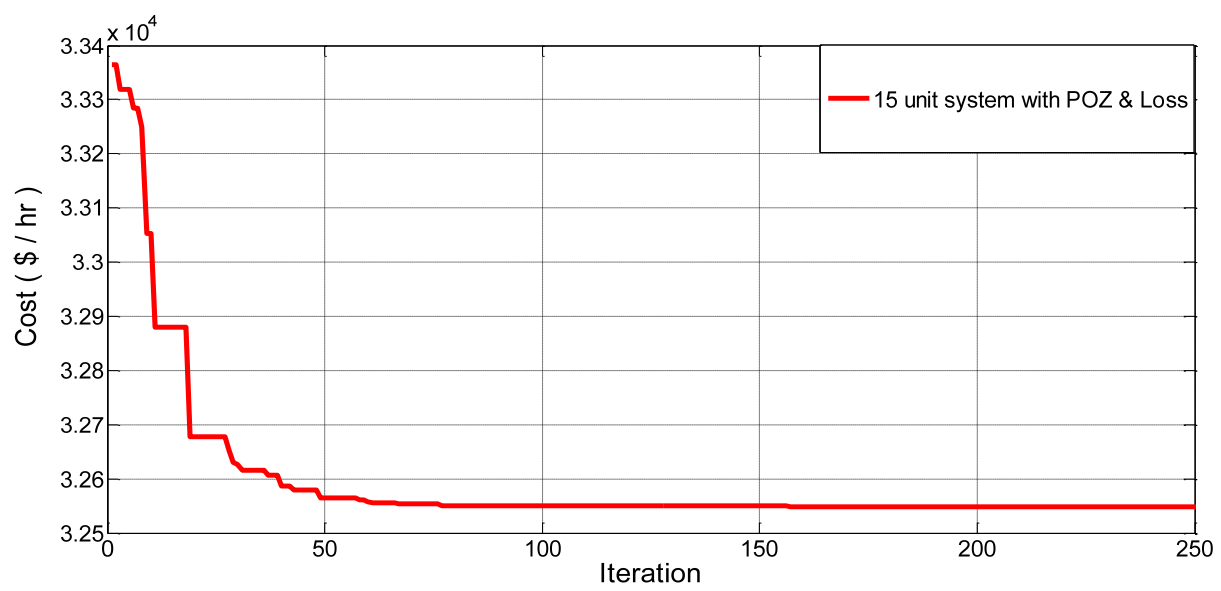

Fig.6. Convergence characteristic of KHAMCD for Test case 1

\section{2) Forty unitnonconvex system}

To examine the superior quality of solution and robustness of KHAMCD algorithm a more realistic test case with valve point loading effect and transmission line losses is included here. The fuel cost coefficient data and transmission line loss coefficients are adopted from [47]. The power demand for system is $10500 \mathrm{MW}$. Solution in terms of optimum generation schedule obtained by simulation is presented in Table 7I. The statistical performance over twenty trials is tabulated in Table 9. The best operating cost achieved by the KHAMCD method is $136446.4053 \$ / \mathrm{hr}$. The comparis on of result has been made with hybrid genetic algorithm with ant colony optimization (GAAPI) [42], shuffled differential evolution (SDE) [43], teaching learning based optimization (TLBO) [44], oppositional real coded chemical reaction optimization (ORCCRO) [45] , krill herd algorithm (KHA) [46], oppositional invasive weed optimization (OIWO) [47] and most recently reported Opposition-based krill herd algorithm (OKHA)[53], hybrid PSO DE approach[54]. Here it can be observed that proposed method KHAMCD is able to achieve cheapest generation cost as compared to other reported methods. Smooth and stable convergence characteristic of this system obtained by KHAMCD algorithm for power demand of $10500 \mathrm{MW}$ with transmission loss is shown in Fig 7.

I.J. Intelligent Systems and Applications, 2016, 11, 34-50 
Table 8. Optimum Generation of KHAMCD for Test Case 2

\begin{tabular}{|c|c|c|c|c|c|c|c|c|c|}
\hline unit & KHAMCD & KH IV[46 ] & OIWO[47] & SDE [43] & unit & KHAMCD & KH IV[46 ] & OIWO[47] & SDE [43] \\
\hline $\mathrm{P} 1$ & 114 & 114 & 113.9908 & 110.06 & $\mathrm{P} 21$ & 523.2794 & 524.4678 & 549.9412 & 544.81 \\
\hline $\mathrm{P} 2$ & 114 & 114 & 114.0000 & 112.41 & $\mathrm{P} 22$ & 535.9596 & 535.5799 & 549.9999 & 550 \\
\hline P3 & 120 & 120 & 119.9977 & 120 & P23 & 523.2794 & 523.3795 & 523.2804 & 550 \\
\hline $\mathrm{P} 4$ & 179.7331 & 190 & 182.5131 & 188.72 & $\mathrm{P} 24$ & 523.2794 & 523.15527 & 523.3213 & 528.16 \\
\hline P5 & 87.8005 & 88.5944 & 88.4227 & 85.91 & P25 & 523.2794 & 524.1916 & 523.5804 & 524.16 \\
\hline P6 & 140 & 105.5166 & 140.0000 & 140 & P26 & 523.2794 & 523.5453 & 523.5847 & 539.10 \\
\hline $\mathrm{P} 7$ & 300 & 300 & 299.9999 & 250.19 & $\mathrm{P} 27$ & 10 & 10.1245 & 10.0086 & 10 \\
\hline P8 & 300 & 300 & 292.0654 & 290.68 & P28 & 10 & 10.1815 & 10.0068 & 10.37 \\
\hline P9 & 300 & 300 & 299.8817 & 300 & P29 & 10 & 10.0229 & 10.0123 & 10 \\
\hline P10 & 279.5997 & 280.6777 & 279.7073 & 282.01 & P30 & 87.7999 & 87.8154 & 87.8664 & 96.10 \\
\hline P11 & 168.7998 & 243.5399 & 168.8149 & 180.82 & P31 & 190 & 190 & 190.0000 & 185.33 \\
\hline P12 & 94 & 168.8017 & 94.0000 & 168.74 & P32 & 190 & 190 & 189.9983 & 189.54 \\
\hline P13 & 484.0392 & 484.1198 & 484.0758 & 469.96 & P33 & 190 & 190 & 190.0000 & 189.96 \\
\hline P14 & 484.0392 & 484.1662 & 484.0477 & 484.17 & P34 & 200 & 200 & 199.9940 & 199.90 \\
\hline P15 & 484.0392 & 485.2375 & 484.0396 & 487.73 & P35 & 200 & 164.9199 & 200.0000 & 196.25 \\
\hline P16 & 484.0392 & 485.0698 & 484.0886 & 482.30 & $\mathrm{P} 36$ & 164.7999 & 164.9787 & 164.8283 & 185.85 \\
\hline P17 & 489.2794 & 489.4539 & 489.2813 & 499.64 & P37 & 110 & 110 & 110.0000 & 109.72 \\
\hline P18 & 489.2794 & 489.3035 & 489.2966 & 411.32 & P38 & 110 & 110 & 109.9940 & 110 \\
\hline P19 & 511.2794 & 510.7127 & 511.3219 & 510.47 & P39 & 110 & 110 & 110.0000 & 95.71 \\
\hline P20 & 550 & 511.3040 & 511.3350 & 542.04 & $\mathrm{P} 40$ & 550 & 512.0677 & 550.0000 & 532.43 \\
\hline \multicolumn{6}{|c|}{ Total cost $(\$ / \mathrm{h})$} & 136446.4053 & 136670.3701 & $136,452.677$ & $138,157.46$ \\
\hline \multicolumn{6}{|c|}{ P loss (MW) } & 958.8845 & 978.9251 & 957.2965 & 974.43 \\
\hline
\end{tabular}

Table 9. Statistical results of KHAMCD for Test Case 2

\begin{tabular}{|c|c|c|c|c|c|}
\hline Method & $\operatorname{Min} \operatorname{Cost}(\$ / \mathrm{hr})$ & Mean $\operatorname{Cost}(\$ / \mathrm{hr})$ & $\operatorname{Max} \operatorname{Cost}(\$ / h r)$ & S.D & CPU time (sec) \\
\hline GAAPI [42] & 139864.96 & NA & NA & NA & NA \\
\hline SDE [43] & $138,157.46$ & NA & NA & NA & NA \\
\hline ORCCRO[45 ] & $136,855.19$ & $136,855.19$ & $136,855.19$ & NA & 14 \\
\hline TLBO[44] & 137814.17 & NA & NA & ------- & ------- \\
\hline QOTLBO[44] & 137329.86 & NA & NA & ------- & ------- \\
\hline OIWO [47 ] & $136,452.68$ & $136,452.68$ & $136,452.68$ & NA & 10.7 \\
\hline KHA-IV[46 ] & 136670.3701 & 136671.2293 & 136671.8648 & NA & NA \\
\hline OKHA[53] & $136,575.97$ & $136,576.15$ & $136,576.64$ & NA & NA \\
\hline KHAMCD & 136446.4053 & 136454.8868 & 136474.1348 & 11.0218 & 9.964 \\
\hline
\end{tabular}




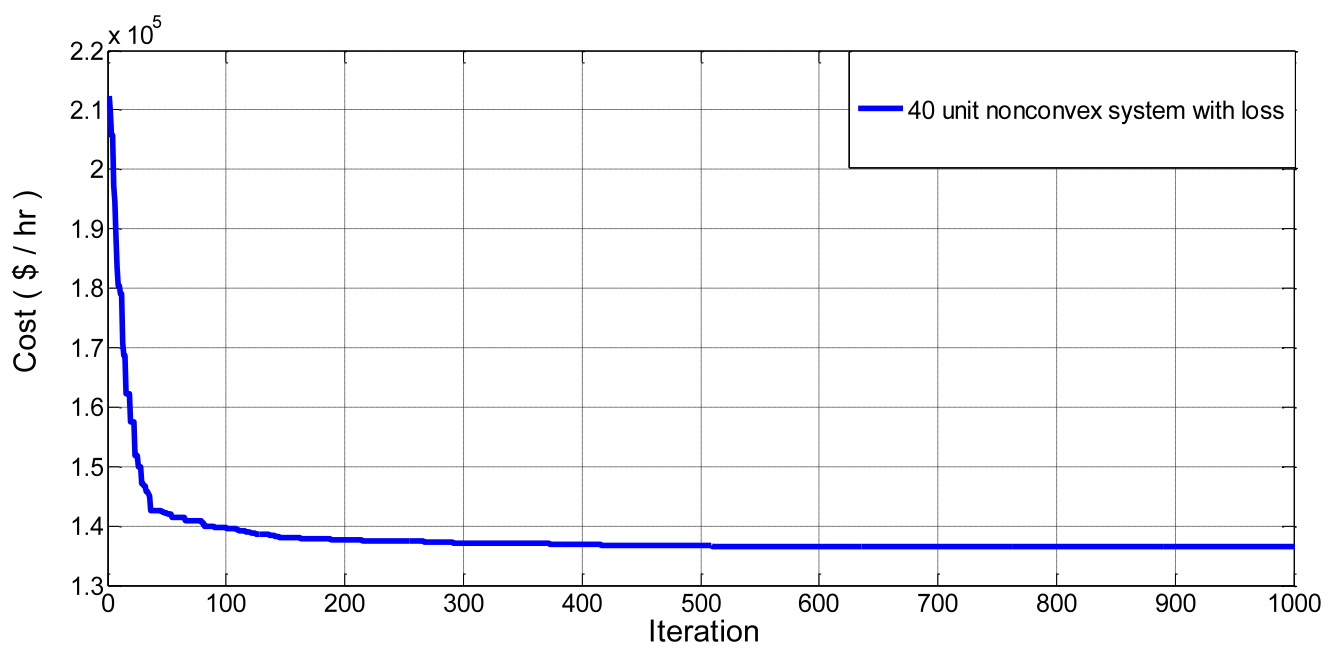

Fig.7. Convergence characteristic of KHAMCD for Test case 2

\section{CONCLUSION}

Krill herd algorithm (KHA) belongs to the family of nature inspired stochastic search algorith ms. To improve global search capability and convergence characteristics, the basic KHA has been improved by (i) adding crossover and mutation operators (KHAMC) and (ii) by including neighborhood distance concept (KHAMCD). The modified versions of KHA are employed to solve eight unimodal/multimodal benchmark functions as well as two non-smooth and non-convex ELD problems of power system. The basis KHA was tested on different mathematical benchmark problems and its performance was found to be satisfactory in terms of convergence and consistency. The performance of the algorith $m$ was found to be improved in terms of solution quality by using mutation and crossover operators. When neighborhood distance concept was added, for the same accuracy, the computational time was reduced to around 26 to $30 \%$ and exploration and exploitation level of krill herd is balanced properly. Various trials were conducted with different initial populations and it was found that every time KHAMCD produced accurate results within tolerance band. As compared to recently reported results in literature, the performance of KHAMCD is found to better in terms of solution quality. From this comparative analysis, it can be concluded that the proposed methodology can effectively be used to solve smooth as well as non-smooth constrained optimization problems.

\section{REFERENCES}

[1] Holland J H. Genetic algorithms and the optimal allocation of trials. SIAM J. Comput., 1973, 2(2): 88-105.

[2] Beyer H G, Schwefel H P. Evolution Strategies: A Comprehensive Introduction. Journal Natural Computing, 2002, 1 (1): 3-52.

[3] Fogel L J, Owens A J, Walsh M J. Artificial intelligence through simulated evolution. 1966, Wiley, New York.

[4] Reynolds R G. An introduction to cultural algorithms. In Proceedings of the 3 rd annual conference on evolutionary programming, 1994. 131-139.

[5] Kirkpatrick S, Gelatt C D, Vecchi M P. Optimization by simulated annealing. Science, 1983, 220(4598):671-680.

[6] Glover F. Tabu search. Part I. ORSA Journal on Computing, 1989, 1(3):190-206.

[7] Kennedy J, Eberhart R C. Particle swarm optimization. In Proceedings of IEEE international conference on neural networks, Piscataway, NJ, 1995. 1942-1948.

[8] Dorigo M, Maniezzo V, Colorni A. The ant system: optimization by a colony of cooperating agents. IEEE Trans. Syst. Man Cybern. Part B: Cybern., 1996, 26:2941.

[9] Geem Z W, Kim J H, Loganathan G. A new heuristic optimization algorithm: harmony search. Simulation, 2001,76(2): 60-68.

[10] Ocenasek J, Schwarz J. Estimation of distribution algorithm for mixed continuous-discrete optimization problems. In 2nd Euro-International Symposium on Computational Intelligence, 2002. 227-232.

[11] Passino K M. Biomimicry of bacterial foraging for distributed optimization and control. IEEE Control Syst. Mag., 2002, 22(3): 52-67.

[12] Haddad O B, Afshar A, Marin o M A. Honey bees mating optimization algorithm (HBMO): a new heuristic approach for en gineering optimization. Proceeding of the First International Conference on Modeling, Simulation and Applied Optimization, 2005.

[13] Shah-Hosseini H. The intelligent water drops algorithm: A nature-inspired swarm-based optimization algorithm. International Journal of Bio-Inspired Computation, 2009, 1(1/2): 71-79.

[14] Yang X-S. Firefly algorithms for multimodal optimization. Stochastic Algorithms, Foundations and Applications. Springer, 2009. 169-178.

[15] Karaboga D, Basturk B. A powerful and efficient algorithm for numerical function optimization: artificial bee colony (ABC) algorithm. J Glob Optim, 2007, 39: 459-471.

[16] Rashedi E, N-pour H, Sary azdi S. GSA: A Gravitational search algorithm. Information Sciences, 2009, 179: 22322248.

[17] Yang X-S. A new metaheuristic bat-inspired algorithm. In 
J. R. Gonzalez et al. (Eds.), Nature inspired cooperative strategies for optimization (NISCO 2010). Studies in computational intelligence, Berlin:. Springer. 2010, 284: 65-74.

[18] Rajabioun R. Cuckoo optimization algorithm. Applied Sof Computing, 2011,11: 5508-5518.

[19] Gandomi A H, Alavi A H. Krill Herd: A New BioInspired Optimization Algorithm. Communications In Nonlinear Science And Numerical Simulation, 2012, 17 : 4831-4845.

[20] Cuevas E, Cienfuegos M. A new algorithm inspired in the behavior of the social-spider for constrained optimization. Expert Sy stems with Applications, 2014, 41: 412-425.

[21] Civicioglu P. Backtracking search optimization algorithm for numerical optimization problems. Applied Mathematics and computation, 2013, 219(15): 8121-8144.

[22] Mirjalili S, Mirjalili S M, Lewis A. Grey Wolf Optimizer. Advances in Engineering Software, 2014, 69: 46-61.

[23] Cheng M-Y, Prayogo D. Symbiotic Organism Search: A metaheuristic optimization algorithm. Computers and Structures, 2014, 139: 98-112.

[24] Yazdani M, Jolai F. Lion optimization algorithm. Journal of Computational Design and Engineering, 2015, doi:10.1016/j.jcde.2015.06.003

[25] Salimi H. Stochastic Fractal search: a powerful metaheuristic algorithm. Knowled ge based systems, 2015 , 75: 1-18.

[26] Shareef H, Ibrahim A A, Mutlag A H. Lightning search algorithm[J]. Applied Soft Computing, 2015, 36: 315-333.

[27] Wolpert D H, Macready W G. No free lunch theorems for optimization . IEEE Trans. Evol. Comput., 1997, 1 (1): 67-82,1997.

[28] Marr J W S. The natural history and geography of the Antarctic krill (Euphausia superba Dana). Disc Rep, 1962, 32:33-464.

[29] Wang G-G, Deb S, Gandomi A H, Alavi A H. Opposition based krill herd algorithm with Cauchy mutation and position clamping. Neurocomputing, 2015, dx.doi.org/10.1016.

[30] Nikbakht H, Mirvaziri H. A new clustering approach on $\mathrm{K}$-means and krill herd algorithm. 23rd Iranian conference on Electrical Engineering, 2015. 662-667.

[31] Pandey S, Patidar R, George N V. Design of a krill herd algorithm based adaptive channel equalizer. International Symposium on Intelligent Signal Processing and Communication System, 2014. 257-260.

[32] Saremi S, Mirjalili S M, Mirjalili S. Chaotic Krill Herd Optimization Algorithm. Procedia Technology, 2014, 12: 180-185.

[33] Bidar M, Fattahi E, Kanan H R. Modified krill herd optimization algorithm using chaotic parameters. 4th International Conference on Computer and Knowledge Engineering, 2014. 420-424.

[34] Wang G-G, Gandomi A H, Alavi A H. Stud krill herd algorithm. Neurocomputing, 2014, 128: 363-370.

[35] Wang G-G, Gandomi A H, Alavi A H. An effective krill herd algorithm with migration operator in biogeography based optimization. Applied Mathematical Modeling, 2014, 38: 2454-2462.

[36] Fattahi E, Bidar M, Kanan H R. Fuzzy krill herd optimization algorithm. IEEE first International Conference on Networks and Soft Computing, 2014. 423426.
[37] Rodrigues D, Pereira L A M, Papa P J, Weber S A T. A Binary krill herd approach for feature selection. 22nd International Conference on Pattern Recognition, 2014. 1407-1412.

[38] Li J, Tang Y, Hua C, Guan X. An improved krill algorithm: Krill herd with linear decreasing step. Applied Mathematics and Computation, 2014, 234: 356-367.

[39] Jamil M, Yang X-S. A literature survey of benchmark functions for global optimization problems. Int. Journal of Mathematical Modeling and numerical optimization, 2013, 4: 150-194.

[40] Dubey H M, Panigrahi B K, Pandit M. Bio-inspired optimization for economic load dispatch: a review, International Journal of Bio-Inspired Computation 2014, 6 (1), 7-21.

[41] Wood A J, Wallenberg B F. Power Generation, Operation and Control. 1984, New York: Wiley.

[42] Ciornei I, Kyriakides E. A GA-API Solution for the Economic Dispatch of Generation in Power System Operation. IEEE Trans Power Sy st., 2012, 27 (1).

[43] Reddy A S, Vaisakh K. Shuffled differential evolution for large scale economic dispatch. Electric Power Systems Research, 2013, 96: 237-45, .

[44] Roy P K, Bhui S. Multi-objective quasi-oppositional teaching learning based optimization for economic emission load dispatch problem. Int. J. Electr. Power Energy Syst., 2013, 53: 937-48.

[45] Bhattacharjee K, Bhattacharya A, Halder nee Dey S. Oppositional Real Coded Chemical Reaction Optimization for different economic dispatch problems. Electrical Power and Energy Systems, 2014, 55: 378-391.

[46] Mandal B, Roy P K, Mandal S. Economic load dispatch using krill herd algorithm. Electrical Power and Energy Systems, 2014, 57: 1-10.

[47] Barisal A K, Prusty R C. Large scale economic dispatch of power systems using oppositional invasive weed optimization. Applied Soft Computing, 2015, 29: 122 137.

[48] Dubey H M, Pandit M, Panigrahi B K, Udgir M. Economic load dispatch by hybrid swarm intelligence based gravitational search algorithm. I J Intelligent Systems and Applications, 2013, 5(8): 21-32.

[49] Dubey H M, Pandit M, Panigrahi B K. A biologically inspired modified flower pollination algorithm for solving economic dispatch problems in modern power systems. Cognitive Computation, 2015, 7(5): 594-608.

[50] Mahdad B, Srairi K.. Solving Practical Economic Dispatch Problems Using Improved Artificial B ee Colony Method. IJJ. Intelligent Systems and Applications, 2014 , 07(6): 36-43.

[51] Labbi Y, Attous D B, Gabbar H A, Mahdad B, Zidan A. A new rooted tree optimization algorithm for economic dispatch with valve-point effect. Electrical Power and Energy Systems 79 (2016) 298-311.

[52] D. Bisen, H. M. Dubey, M. Pandit, B.K. Panigrahi, solution of large scale economic load dispatch problem using quadratic programming and GAMS: A comparative analysis, Journal of Information and Computing science, vol. 7,no.3,pp.200-2011,2012.

[53] Ali Bulbul S M, Pradhan M, Roy P K, T. Pal. Oppositionbased krill herd algorithm applied to economic load dispatch problem. Ain Shams Engineering Journal, .doi.org/10.1016/j.asej.2016.02.003 
[54] Parouha R P, Das K N. A novel hybrid optimizer for solving Economic Load Dispatch problem. Electrical Power and Energy Systems, 2016, 78:108-126.

[55] Singh G P, .Singh A. Comparative Study of Krill Herd, Firefly and Cuckoo Search Algorithms for Unimodal and Multimodal Optimization. I.J. Intelligent Systems and Applications, 2014, 03:35-49

\section{Authors' Profiles}

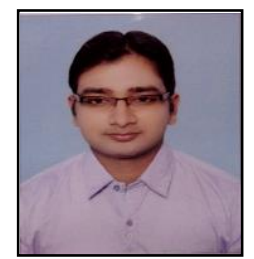

Prasun K. Agrawal obtained his B.Tech degree in Electrical and Electronics Engineering from Integral University, Lucknow, (India) in 2013. He is currently pursuing his M.E degree in Electrical Engineering (Industrial Sy stem and Drives), from M.I.T.S, Gwalior, (India). His areas of interest are Optimal Power Flow, evolutionary computing and artificial intelligence applications to Power System.

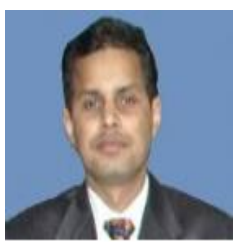

Hari Mohan Dubey obtained his M.E. degree in Electrical Engineering from Madhav Institute of Technology \& Science Gwalior (India) in 2002. He is currently working as Assistant Professor in Department of Electrical Engineering, M.I.T.S., Gwalior, (India). His areas of research are Computational intelligence algorithm and their applications to power system.

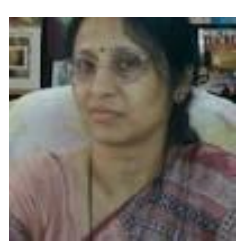

Manjaree Pandit obtained her M. Tech degree in Electrical Engineering from Maulana Azad College of Technology, Bhopal, (India) in 1989 and Ph.D. degree in 2001. She is currently working as Professor in Department of Electrical Engineering, M.I.T.S., Gwalior, (India). Her areas of interest are Power System Security Analysis, Optimization using soft computing/ evolutionary methods, ANN and Fuzzy neural ap plications to Power System.

How to cite this paper: Prasun Kumar Agrawal, Manjaree Pandit, Hari Mohan Dubey, "Improved Krill Herd Algorithm with Neighborhood Distance Concept for Optimization", International Journal of Intelligent Systems and Applications (IJISA), Vol.8, No.11, pp.34-50, 2016. DOI: 10.5815/ijisa.2016.11.05 